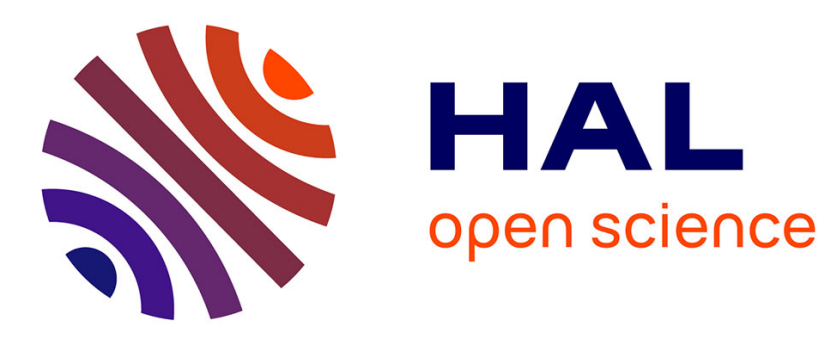

\title{
Synthesis and Evaluation of Antitumor Alkylphospholipid Prodrugs
}

Boris Gaillard, Jean-Serge Remy, Françoise Pons, Luc Lebeau

\section{To cite this version:}

Boris Gaillard, Jean-Serge Remy, Françoise Pons, Luc Lebeau. Synthesis and Evaluation of Antitumor Alkylphospholipid Prodrugs. Pharmaceutical Research, 2020, 37 (6), pp.106. 10.1007/s11095-02002830-y . hal-02990890

\section{HAL Id: hal-02990890 https://hal.science/hal-02990890}

Submitted on 25 Nov 2020

HAL is a multi-disciplinary open access archive for the deposit and dissemination of scientific research documents, whether they are published or not. The documents may come from teaching and research institutions in France or abroad, or from public or private research centers.
L'archive ouverte pluridisciplinaire HAL, est destinée au dépôt et à la diffusion de documents scientifiques de niveau recherche, publiés ou non, émanant des établissements d'enseignement et de recherche français ou étrangers, des laboratoires publics ou privés. 


\title{
Synthesis and Evaluation of Antitumor Alkylphospholipid Prodrugs
}

Boris Gaillard, Jean-Serge Remy, Françoise Pons, and Luc Lebeau ${ }^{1}$

Laboratoire de Conception et Application de Molécules Bioactives,

UMR 7199 CNRS - Université de Strasbourg,

Faculté de Pharmacie, 74 route du Rhin - BP 60024, 67401 Illkirch, France.

\begin{abstract}
Purpose Hemolysis is a serious side effect of antitumor alkylphospholipids (APLs) that limits dose levels and is a constraint in their use in therapeutic regimen. Nine prodrugs of promising APLs (miltefosine, perifosine, and erufosine) were synthesized so as to decrease their membrane activity and improve their toxicity profile while preserving their antineoplastic potency.
\end{abstract}

Methods The synthesis of the pro-APLs was straightforwardly achieved in one step starting from the parent APLs. The critical aggregation concentration of the prodrugs, their hydrolytic stability under various $\mathrm{pH}$ conditions, their blood compatibility and cytotoxicity in three different cell lines were determined and compared to those of the parent antitumor lipids.

Results The APL prodrugs display antitumor activity which is similar to that of the parent alkylphospholipids but without associated hemolytic toxicity.

Conclusion The pro-APL compounds may be considered as intravenously injectable derivatives of APLs. They could thus address one of the major issues met in cancer therapies involving antitumor lipids and restricting their utilization to oral and topical administration because of limited maximum tolerated dose.

KEYWORDS miltefosine, perifosine, erufosine, alkylphospholipid, prodrug, hemolytic toxicity

\footnotetext{
${ }^{1}$ Corresponding author: 1lebeau@,unistra.fr; Tel: +33 (0)3 688543 03; Fax: +33 (0)3 68854306.
} 


\section{INTRODUCTION}

Lysophosphatidylcholines (lysoPCs) are endogenous cell membrane components produced from phosphatidylcholines by phospholipases. They act as modulators of several signaling pathways and biochemical routes. Since these compounds are not stable and are substrates for acyltransferases and lysophospholipases, efforts have been made to develop alkylphospholipids (APLs) as metabolically stable synthetic analogs of lysoPCs for translational health research and clinical trials. Some of these analogs revealed effective immune modulating properties but also demonstrated selective antineoplastic activities (1). This novel class of antitumor agents thus served as the basis for the synthesis of a wider diversity of structural variants that have been reviewed elsewhere (2). Among them, miltefosine, perifosine, and erufosine proved especially promising and have been tested in phase I and phase II clinical trials for their antitumor activity (Fig. 1).

Miltefosine has been described for the first time by Eibl and Unger in 1987 (3). It has been evaluated in vitro (4) and in vivo (5), and its clinical development for oral treatment of patients with solid tumors was prevented by dose-limiting gastrointestinal toxicity (6). The intravenous (iv) route being excluded because of high hemolytic activity (7), miltefosine is currently used for topical administration. Perifosine results from pharmacomodulation at the headgroup and was developed in order to get improved biological stability so as to expand the therapeutic range of the compound (8). Replacement of the choline substituent by the 4hydroxy- $N, N$-dimethylpiperidinium moiety and elongation of the hydrophobic chain to 18 carbon atoms were assumed to lead to a less toxic compound, while preserving the antineoplastic potency. These expectations have been fulfilled to a large extent and phase I and phase II clinical trials were conducted (9). However, whereas perifosine had extended half-life in plasma (137 h vs. $96 \mathrm{~h}$ for miltefosine) (10), it was associated with high hemolytic activity (11). Oral administration caused toxicity that was similar to that of miltefosine and 
the treatment of various cancers proved unsatisfactory (12-14). Consequently, perifosine cannot be used as monotherapy but provides interesting results in combination with radiotherapy (15). Erufosine is a member of the third generation of APLs. It combines an $\omega-9$ cis-unsaturated alkyl chain with 22 carbon atoms and a homophosphocholine moiety. Extension of the hydrophobic alkyl chain, as compared to the previous APLs, results in a decrease in aqueous solubility of the compound which organizes into lamellar structures instead of micelles (16). The critical micellar concentration (CMC) for such compounds was thus evaluated in the range of $10^{-7}-10^{-8} \mathrm{M}$ which is 2 to 3 orders of magnitude below that for miltefosine $\left(10^{-5} \mathrm{M}\right)(17)$, suggesting that intravenous administration can be considered. Favorable pharmacokinetic and pharmacodynamic properties such as potent cytotoxicity, low gastrointestinal toxicity and reduced myelotoxic and hemolytic effects were found $(18,19)$. Erufosine has been involved in multiple cell-based and preclinical studies (20) and phase I clinical trials are expected.

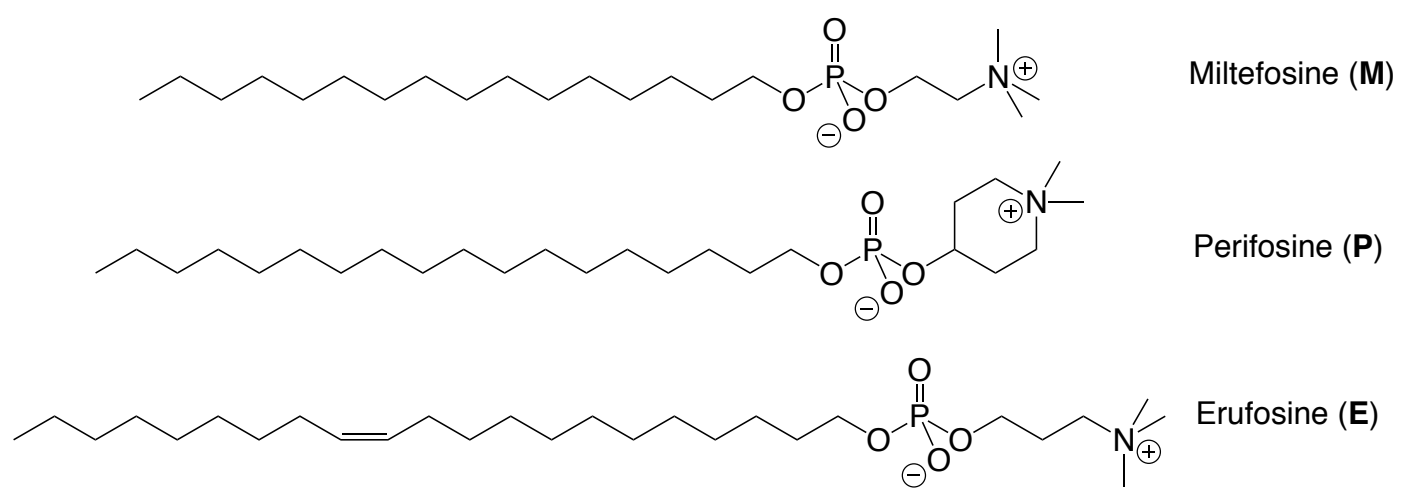

Figure 1 Structure of the three APLs considered in this work.

APLs offer an advantage over conventional DNA-interacting chemotherapeutic agents as they have a unique mode of action on cell membranes. However, the clinical development of these compounds is seriously restricted by their intrinsic surface active properties that translate into unfavorable pharmacokinetics and pharmacodynamics. This prompted us to investigate prodrugs of APLs to address this issue. Searching carefully in the literature, we 
identified only very few derivatives of APLs that could be potential prodrugs (Fig. 2). The first one (PDФРC) was developed in McDonald's group in 2006 and was formally the result of the esterification of miltefosine with a branched diacylglyceride (21). Two other compounds described by Øpstad et al. consist in conjugates between miltefosine and polyenoic chromophores, retinoic and carotenoic acids, through a glycol linker (M-Car and M-Ret, resp.) (22). These 3 compounds carry a net positive charge and were designed as DNA transfer reagents. Whether they can regenerate antitumor miltefosine when exposed to degradation enzymes in a biological environment was not investigated by the authors. Starting from our previous work on DOPC-based biolabile gene transfer reagents (23-25), we have designed prodrugs of APLs (pro-APLs) and examined their intrinsic physicochemical properties. This has been realized through determination of their hydrolytic stability as a function of $\mathrm{pH}$, and analysis of their surface active and aggregation properties. The antitumor
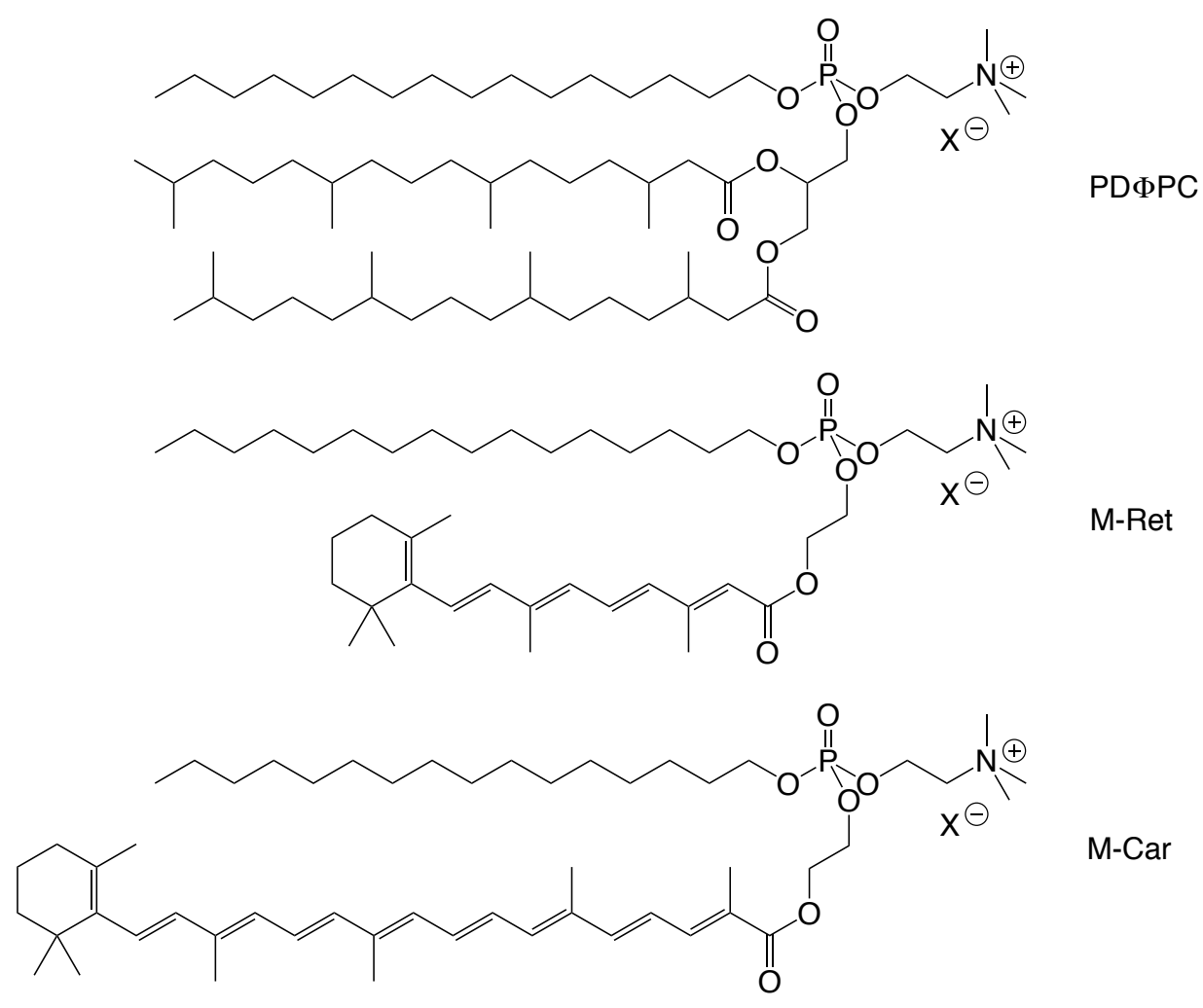

Figure 2 Structure of the three miltefosine derivatives described in the literature and developed for gene delivery purposes $(21,22)$. 
activity of the pro-APLs in various normal and tumor cell lines was characterized by $\mathrm{IC}_{50}$ determination, and blood compatibility was investigated. Whereas compounds retained full antitumor activity of the parent APLs, they did not reveal harmful to erythrocytes and did not provoke hemolysis, the dose-limiting side-effect associated with APLs.

\section{MATERIALS AND METHODS}

\section{Materials}

General information on materials can be found as Supplementary Information. The preparation of erufosine and $\mathbf{E}_{\mathbf{a}-\mathbf{c}}$ prodrugs has been described elsewhere (26).

Abbreviations: PE, petroleum ether; s, singlet; d, doublet; t, triplet; q, quadruplet; b, broad.

\section{Miltefosine (M)}

Triethylamine $(822 \mu \mathrm{L}, 5.90 \mathrm{mmol})$ was slowly added to freshly distilled $\mathrm{POCl}_{3}(500 \mu \mathrm{L}$, $5.36 \mathrm{mmol})$ in dry $\mathrm{THF}(20 \mathrm{~mL})$ at $0{ }^{\circ} \mathrm{C}$ under argon. Then 1-hexadecanol $(1.30 \mathrm{~g}$, $5.36 \mathrm{mmol})$ in THF $(10 \mathrm{~mL})$ was added over $30 \mathrm{~min}$ and the resulting mixture was warmed to rt. When all the alcohol had reacted (checked by TLC), the mixture was cooled to $0{ }^{\circ} \mathrm{C}$ and a second portion of triethylamine $(3.3 \mathrm{~mL}, 21.4 \mathrm{mmol})$ was added, followed by 2-bromoethanol $(380 \mu \mathrm{L}, 5.36 \mathrm{mmol})$ in THF $(10 \mathrm{~mL})$. The reaction mixture was stirred overnight at $\mathrm{rt}$, decomposed with $\mathrm{HCl} 10 \%(10 \mathrm{~mL})$ and heated at $40{ }^{\circ} \mathrm{C}$ for $2 \mathrm{~h}$. THF was removed under vacuum and the aqueous residue was extracted with $\mathrm{CH}_{2} \mathrm{Cl}_{2}$. The organic layer was dried over $\mathrm{Na}_{2} \mathrm{SO}_{4}$, filtered, reduced under vacuum, and the crude residue was purified by flash chromatography $\left(\mathrm{CH}_{2} \mathrm{Cl}_{2} / \mathrm{MeOH} 10: 0\right.$ to $\left.8: 2\right)$ to yield intermediate 2-bromoethyl hexadecyl hydrogenophosphate $(1.01 \mathrm{~g}, 44 \%) . \quad R_{\mathbf{f}}: 0.43 \quad\left(\mathrm{CH}_{2} \mathrm{Cl}_{2} / \mathrm{MeOH} / \mathrm{H}_{2} \mathrm{O} \quad 75: 22: 3\right) .{ }^{1} \mathrm{H}-\mathrm{NMR}$ $\left(400 \mathrm{MHz}, \mathrm{MeOD} / \mathrm{CDCl}_{3} 1: 1\right) \delta 0.85(\mathrm{t}, J=6.7 \mathrm{~Hz}, 3 \mathrm{H}) ; 1.24(\mathrm{~m}, 26 \mathrm{H}) ; 1.60\left(\mathrm{tt}, J_{l}=J_{2}=6.4 \mathrm{~Hz}\right.$, $2 \mathrm{H}) ; 3.51(\mathrm{t}, J=6.4 \mathrm{~Hz} ; 2 \mathrm{H}) ; 3.86\left(\mathrm{td}, J_{l}=J_{2}=6.5 \mathrm{~Hz}, 2 \mathrm{H}\right) ; 4.10\left(\mathrm{t}, J_{l}=J_{2}=6.5 \mathrm{~Hz}, 2 \mathrm{H}\right)$. 
${ }^{13} \mathrm{C}-\mathrm{NMR}\left(100.7 \mathrm{MHz}, \mathrm{MeOD} / \mathrm{CDCl}_{3} 1: 1\right) \delta 14.3 ; 23.3 ; 26.4 ; 30.0 ; 30.3$ (br); 31.3; 32.6; 66.0; 66.7. ${ }^{31} \mathrm{P}-\mathrm{NMR}\left(162 \mathrm{MHz} ; \mathrm{MeOD} / \mathrm{CDCl}_{3} 1: 1\right) \delta-0.56$. IR (ATR) $v$ 495; 596; 695; 733; $772 ; 879 ; 1007 ; 1218 ; 1270 ; 1456 ; 2852 ; 2921$.

The previous compound (1.0 g, $2.33 \mathrm{mmol})$ in $\mathrm{CHCl}_{3} / \mathrm{CH}_{3} \mathrm{CN} / i-\mathrm{PrOH}$ 3:5:5 (13 mL) was treated with $45 \%(\mathrm{w} / \mathrm{w})$ aqueous $\mathrm{NMe}_{3}(5 \mathrm{~mL}, 34.3 \mathrm{mmol})$ at $70{ }^{\circ} \mathrm{C}$. When all starting material was consumed (checked by TLC), volatile was removed under vacuum and the residue was extracted with $\mathrm{CHCl}_{3} / \mathrm{MeOH}$ 1:3. The organic layer was washed with sat. $\mathrm{NaCl}$, reduced under vacuum, diluted with $\mathrm{CHCl}_{3}$, dried over $\mathrm{MgSO}_{4}$, filtered and evaporated. The crude residue was purified by flash chromatography $\left(\mathrm{CH}_{2} \mathrm{Cl}_{2} / \mathrm{MeOH} / \mathrm{H}_{2} \mathrm{O}\right.$ 75:22:3 to 45:45:10) to yield miltefosine (0.77 g, 81\%). $R_{\mathrm{f}}: 0.1 \quad\left(\mathrm{CH}_{2} \mathrm{Cl}_{2} / \mathrm{MeOH} / \mathrm{H}_{2} \mathrm{O} \quad 75: 22: 3\right)$. ${ }^{1} \mathrm{H}-\mathrm{NMR}\left(400 \mathrm{MHz}, \mathrm{MeOD} / \mathrm{CDCl}_{3} 1: 1\right) \delta 0.85(\mathrm{t}, J=6.2 \mathrm{~Hz}, 3 \mathrm{H}) ; 1.24(\mathrm{~m}, 26 \mathrm{H}) ; 1.61$ (tt, $\left.J_{l}=J_{2}=6.4 \mathrm{~Hz}, 2 \mathrm{H}\right) ; 3.31(\mathrm{~s}, 9 \mathrm{H}) ; 3.62(\mathrm{~m}, 2 \mathrm{H}) ; 3.85\left(\mathrm{td}, J_{1}=J_{2}=6.5 \mathrm{~Hz}, 2 \mathrm{H}\right) ; 4.23(\mathrm{~m}$, 2H). ${ }^{13} \mathrm{C}-\mathrm{NMR}\left(100.7 \mathrm{MHz}, \mathrm{MeOD} / \mathrm{CDCl}_{3} 1 / 1\right) \delta 14.3 ; 23.2 ; 26.4 ; 30.1$ (2C); 30.3 (8C); 31.3; 32.5; 54.5; 59.7; 66.7; 67.0. ${ }^{31} \mathrm{P}-\mathrm{NMR}\left(162 \mathrm{MHz} ; \mathrm{MeOD} / \mathrm{CDCl}_{3} 1: 1\right) \delta-0.32$. IR (ATR) v 497; 716; 746; 853; 927; 957; 1050;1126; 1246; 1471; 1633; 2849; 2914; 3372. HR-MS (ESI+) m/z [M+H] $]^{+}$calcd for $\mathrm{C}_{21} \mathrm{H}_{47} \mathrm{NO}_{4} \mathrm{P}^{+}$408.3237, found 408.3239.

\section{Perifosine $(\boldsymbol{P})$}

Phosphorus oxychloride $(864 \mu \mathrm{L}, 9.2 \mathrm{mmol})$ was added to 1-octadecanol (2.50 g, $9.2 \mathrm{mmol})$ in anhydrous $\mathrm{Et}_{2} \mathrm{O}(100 \mathrm{~mL})$ at $0{ }^{\circ} \mathrm{C}$, followed by dropwise addition of triethylamine $(1.29 \mathrm{~mL}, 9.2 \mathrm{mmol})$. The reaction mixture was stirred at $\mathrm{rt}$ for $16 \mathrm{~h}$, filtered and the filtrate was reduced under vacuum. The dry residue was dissolved in anhydrous $\mathrm{CHCl}_{3}(100 \mathrm{~mL})$ and $\mathrm{N}, \mathrm{N}$-dimethyl-4-hydroxypiperidinium tosylate $(2.78 \mathrm{~g}, 9.24 \mathrm{mmol})$, triethylamine $(3.00 \mathrm{~mL}$, $21.5 \mathrm{mmol})$, and 4-DMAP $(65 \mathrm{mg}, 0.53 \mathrm{mmol})$ in $\mathrm{CHCl}_{3}(100 \mathrm{~mL})$ were introduced in the reaction flask and allowed to react for $2 \mathrm{~d}$ at rt. The reaction mixture was then reduced under 
vacuum, suspended in THF $(100 \mathrm{~mL})$ and refluxed with $\mathrm{H}_{2} \mathrm{O}(2 \mathrm{~mL})$ for $6 \mathrm{~h}$. The crude mixture was reduced under vacuum and the residue was directly purified by flash chromatography $\left(\mathrm{CHCl}_{3} / \mathrm{MeOH} / \mathrm{NH}_{4} \mathrm{OH} 28 \%\right.$ 10:6:1) to yield perifosine $(0.70 \mathrm{~g}, 16 \%) . R_{\mathrm{f}}$ : $0.2\left(\mathrm{CH}_{2} \mathrm{Cl}_{2} / \mathrm{MeOH} / \mathrm{H}_{2} \mathrm{O}\right.$ 75:22:3). ${ }^{1} \mathrm{H}-\mathrm{NMR}\left(400 \mathrm{MHz}, \mathrm{MeOD} / \mathrm{CDCl}_{3} 1: 1\right) \quad \delta \quad 0.85$ (t, $J=6.5 \mathrm{~Hz}, 3 \mathrm{H}) ; 1.24(\mathrm{~m}, 30 \mathrm{H}) ; 1.59(\mathrm{~m}, 2 \mathrm{H}) ; 2.10(\mathrm{~m}, 4 \mathrm{H}) ; 3.09(\mathrm{~s}, 3 \mathrm{H}) ; 3.16(\mathrm{~s}, 3 \mathrm{H}) ; 3.25-$ $3.53(\mathrm{~m}, 4 \mathrm{H}) ; 3.82(\mathrm{~m}, 2 \mathrm{H}) ; 4.40(\mathrm{~m}, 1 \mathrm{H}) .{ }^{13} \mathrm{C}-\mathrm{NMR}\left(100.7 \mathrm{MHz}, \mathrm{MeOD} / \mathrm{CDCl}_{3} 1: 1\right) \delta$ 14.3; $23.2 ; 26.4 ; 27.2(2 \mathrm{C}) ; 29.9 ; 30.0 ; 30.2(10 \mathrm{C}) ; 31.5 ; 32.5 ; 55.4(2 \mathrm{C}) ; 59.2(2 \mathrm{C}) ; 65.0 ; 66.6 .{ }^{31} \mathrm{P}-$ NMR (162 MHz; $\left.\mathrm{MeOD} / \mathrm{CDCl}_{3} 1: 1\right) \delta-0.40$. IR $v$ 479; 720; 846; 919; 994; 1062; 1124; $1156 ; 1225 ; 1468 ; 2848 ; 2916$. HR-MS $(\mathrm{ESI}+) \mathrm{m} / \mathrm{z}[\mathrm{M}+\mathrm{Na}]^{+}$calcd for $\mathrm{C}_{25} \mathrm{H}_{52} \mathrm{NNaO}_{4} \mathrm{P}^{+}$ 484.3526, found 484.3528.

\section{General Procedure for the Preparation of pro-APLs}

Pro-APLs were prepared according to a previously reported procedure (26). Briefly, electrophilic reagent and APL were stirred in refluxing dry $\mathrm{CHCl}_{3}$ for $24 \mathrm{~h}$ under argon. Volatile was distilled off and the residue was purified by flash chromatography $\left(\mathrm{CH}_{2} \mathrm{Cl}_{2} / \mathrm{MeOH}\right.$ 10:0 to 7:3) to yield the corresponding pro-APL.

\section{2-(((Dodecyloxy)(hexadecyloxy)phosphoryl)oxy)-N,N,N-trimethylethan-1-aminium triflate} $\left(M_{a}\right)$

Compound $\mathbf{M}_{\mathbf{a}}(100 \mathrm{mg}, 84 \%)$ was obtained from miltefosine $(80 \mathrm{mg}, 0.20 \mathrm{mmol})$ and dodecyl triflate (27) $(162 \mathrm{mg}, 0.53 \mathrm{mmol})$ according to the general procedure except the reaction was conducted at rt. $R_{\mathrm{f}}: 0.7\left(\mathrm{CH}_{2} \mathrm{Cl}_{2} / \mathrm{MeOH} / \mathrm{H}_{2} \mathrm{O}\right.$ 75:22:3). ${ }^{1} \mathrm{H}-\mathrm{NMR}(400 \mathrm{MHz}$, $\left.\mathrm{CDCl}_{3}\right) \delta 0.88(\mathrm{t}, J=6.6 \mathrm{~Hz}, 6 \mathrm{H}) ; 1.26(\mathrm{~m}, 44 \mathrm{H}) ; 1.68\left(\mathrm{tt}, J_{1}=J_{2}=6.8 \mathrm{~Hz}, 4 \mathrm{H}\right) ; 3.31(\mathrm{~s}, 9 \mathrm{H}) ;$ $3.83(\mathrm{~m}, 2 \mathrm{H}) ; 4.08(\mathrm{~m}, 4 \mathrm{H}) ; 4.48(\mathrm{~m}, 2 \mathrm{H}) .{ }^{13} \mathrm{C}-\mathrm{NMR}\left(100.7 \mathrm{MHz}, \mathrm{CDCl}_{3}\right) \delta 14.3(2 \mathrm{C}) ; 22.9$ (2C); 25.6 (2C); 29.4 (2C); 29.6 (2C); 29.7 (2C); 29.8 (4C); 29.9 (6C); 30.5 (2C); 32.1 (2C); 54.6 (3C); 61.2; 65.9; 69.2 (2C). ${ }^{31} \mathrm{P}-\mathrm{NMR}\left(162 \mathrm{MHz}, \mathrm{CDCl}_{3}\right) \delta-2.14 . \mathrm{IR} \vee 517 ; 574 ; 638$; 
$1030 ; 1161 ; 1226 ; 1253 ; 1467 ; 2852 ; 2921 ; 3500$. HR-MS $(\mathrm{ESI}+) \mathrm{m} / \mathrm{z}[\mathrm{M}-\mathrm{Cl}]^{+}$calcd for $\mathrm{C}_{33} \mathrm{H}_{71} \mathrm{NO}_{4} \mathrm{P}^{+}$576.5115, found 576.5108.

\section{2-((((Dodecanoyloxy)methoxy)(hexadecyloxy)phosphoryl)oxy)-N,N,N-trimethylethan-} 1-aminium chloride $\left(\boldsymbol{M}_{\boldsymbol{b}}\right)$

Compound $\mathbf{M}_{\mathbf{b}}$ (136 mg, $42 \%$ ) was obtained from miltefosine (200 mg, $\left.0.49 \mathrm{mmol}\right)$ and chloromethyl dodecanoate (24) (979 mg, $3.94 \mathrm{mmol}$ ) according to the general procedure. $R_{\mathrm{f}}$ : $0.5\left(\mathrm{CH}_{2} \mathrm{Cl}_{2} / \mathrm{MeOH} / \mathrm{H}_{2} \mathrm{O}\right.$ 75:22:3). ${ }^{1} \mathrm{H}-\mathrm{NMR}\left(400 \mathrm{MHz}, \mathrm{MeOD} / \mathrm{CDCl}_{3} 1: 1\right) \quad \delta \quad 0.85$ (t, $J=6.3 \mathrm{~Hz}, 6 \mathrm{H}) ; 1.24(\mathrm{~m}, 42 \mathrm{H}) ; 1.63\left(\mathrm{tt}, J_{l}=J_{2}=6.8 \mathrm{~Hz}, 2 \mathrm{H}\right) ; 1.69\left(\mathrm{tt}, J_{l}=J_{2}=6.6 \mathrm{~Hz}, 2 \mathrm{H}\right)$; $2.40(\mathrm{t}, J=7.5 \mathrm{~Hz}, 2 \mathrm{H}) ; 3.23(\mathrm{~s}, 9 \mathrm{H}) ; 3.76(\mathrm{~m}, 2 \mathrm{H}) ; 4.11\left(\mathrm{td}, J_{l}=J_{2}=6.3 \mathrm{~Hz}, 2 \mathrm{H}\right) ; 4.50(\mathrm{~m}$, 2H); 5.64 (ABX syst., $\left.J_{A B}=5.2 \mathrm{~Hz}, J_{A X}=13.4 \mathrm{~Hz}, J_{B X}=11.4 \mathrm{~Hz}, 2 \mathrm{H}\right) .{ }^{13} \mathrm{C}-\mathrm{NMR}(100.7 \mathrm{MHz}$, $\left.\mathrm{MeOD} / \mathrm{CDCl}_{3} 1: 1\right) \delta 14.5(2 \mathrm{C}) ; 23.3(2 \mathrm{C}) ; 25.1 ; 25.9 ; 29.7 ; 29.8 ; 29.9 ; 30.0(2 \mathrm{C}) ; 30.1$ (4C); 30.2 (7C); 30.7; 32.5; 34.6; 54.7 (3C); 62.3; 66.5; 70.2; 84.2; 173.2. ${ }^{31} \mathrm{P}-\mathrm{NMR}(162 \mathrm{MHz}$, $\left.\mathrm{MeOD} / \mathrm{CDCl}_{3} 1: 1\right) \delta-3.80 . \mathrm{IR} \vee 458 ; 490 ; 721 ; 832 ; 873 ; 965 ; 1042 ; 1159 ; 1264 ; 1468 ;$ 1760; 2849; 2917; 2956; 3382. HR-MS (ESI+) m/z [M-Cl $]^{+}$calcd for $\mathrm{C}_{34} \mathrm{H}_{71} \mathrm{NO}_{6} \mathrm{P}^{+} 620.5014$, found 620.5004 .

\section{2-(((1-(Dodecanoyloxy)ethoxy)(hexadecyloxy)phosphoryl)oxy)-N,N,N-trimethylethan-} 1-aminium chloride $\left(\boldsymbol{M}_{\boldsymbol{c}}\right)$

Compound $\mathbf{M}_{\mathbf{c}}$ (169 mg, $51 \%$ ) was obtained as two separated couples of enantiomers (E1 and E2) from miltefosine $(201 \mathrm{mg}, 0.49 \mathrm{mmol})$ and 1-chloroethyl dodecanoate (24) (1.04 g, $3.95 \mathrm{mmol}$ ) according to the general procedure. $R_{\mathrm{f}}: 0.5$ (E1) and 0.4 (E2) $\left(\mathrm{CH}_{2} \mathrm{Cl}_{2} / \mathrm{MeOH} / \mathrm{H}_{2} \mathrm{O}\right.$ 75:22:3). ${ }^{1} \mathrm{H}-\mathrm{NMR}\left(400 \mathrm{MHz}, \mathrm{MeOD} / \mathrm{CDCl}_{3}\right.$ 1:1) E1 $\delta \quad 0.85$ (t, $J=6.1 \mathrm{~Hz}, 6 \mathrm{H}) ; 1.24(\mathrm{~m}, 42 \mathrm{H}) ; 1.58(\mathrm{~d}, J=5.3 \mathrm{~Hz}, 3 \mathrm{H}) ; 1.61\left(\mathrm{tt}, J_{l}=J_{2}=6.8 \mathrm{~Hz}, 2 \mathrm{H}\right) ; 1.69$ $\left(\mathrm{tt}, J_{1}=J_{2}=6.6 \mathrm{~Hz}, 2 \mathrm{H}\right) ; 2.37(\mathrm{t}, J=7.2 \mathrm{~Hz}, 2 \mathrm{H}) ; 3.24(\mathrm{~s}, 9 \mathrm{H}) ; 3.76(\mathrm{~m}, 2 \mathrm{H}) ; 4.09(\mathrm{td}$, $\left.J_{l}=J_{2}=6.7 \mathrm{~Hz}, 2 \mathrm{H}\right) ; 4.50(\mathrm{~m}, 2 \mathrm{H}) ; 6.42\left(\mathrm{qd}, J_{l}=J_{2}=5.3 \mathrm{~Hz}, 1 \mathrm{H}\right) .{ }^{1} \mathrm{H}-\mathrm{NMR}(400 \mathrm{MHz}$, 
$\left.\mathrm{MeOD} / \mathrm{CDCl}_{3} 1: 1\right)$ E2 $\delta 0.86(\mathrm{t}, J=6.2 \mathrm{~Hz}, 6 \mathrm{H}) ; 1.24(\mathrm{~m}, 42 \mathrm{H}) ; 1.56(\mathrm{~d}, J=5.3 \mathrm{~Hz}, 3 \mathrm{H}) ; 1.61$ $\left(\mathrm{tt}, J_{l}=J_{2}=6.8 \mathrm{~Hz}, 2 \mathrm{H}\right) ; 1.68\left(\mathrm{tt}, J_{l}=J_{2}=6.6 \mathrm{~Hz}, 2 \mathrm{H}\right) ; 2.35(\mathrm{t}, J=7.3 \mathrm{~Hz}, 2 \mathrm{H}) ; 3.24(\mathrm{~s}, 9 \mathrm{H}) ;$ $3.77(\mathrm{~m}, 2 \mathrm{H}) ; 4.09\left(\mathrm{td}, J_{l}=J_{2}=6.3 \mathrm{~Hz}, 2 \mathrm{H}\right) ; 4.48(\mathrm{~m}, 2 \mathrm{H}) ; 6.48\left(\mathrm{qd}, J_{l}=J_{2}=5.3 \mathrm{~Hz}, 2 \mathrm{H}\right)$. ${ }^{13} \mathrm{C}-\mathrm{NMR}\left(100.7 \mathrm{MHz}, \mathrm{MeOD} / \mathrm{CDCl}_{3} 1: 1\right) \mathrm{E} 1 \delta 14.5$ (2C); 21.7; 23.4 (2C); 25.3; 26.1; 29.6; 29.7; 29.8; 29.9; 30.0 (2C); 30.1 (2C); 30.2 (4C); 30.3 (4C); 30.7; 32.5 (2C); 34.5; 54.6 (3C); 62.3; 66.5; 70.0; 92.4; 173.3. ${ }^{13} \mathrm{C}-\mathrm{NMR}\left(100.7 \mathrm{MHz}, \mathrm{MeOD} / \mathrm{CDCl}_{3} 1: 1\right)$ E2 $\delta 14.5(2 \mathrm{C}) ; 21.6$; 23.4 (2C); 25.3; 26.1; 29.6; 29.7; 29.8; 29.9; 30.0 (2C); 30.1 (2C); 30.2 (4C); 30.3 (4C); 30.7; 32.5 (2C); 34.5; 54.6 (3C); 62.0; 66.3; 70.2; 92.3; 172.8. ${ }^{31} \mathrm{P}-\mathrm{NMR}\left(162 \mathrm{MHz}, \mathrm{MeOD} / \mathrm{CDCl}_{3}\right.$ 1:1) E1 $\delta-5.96 .{ }^{31} \mathrm{P}-\mathrm{NMR}\left(162 \mathrm{MHz}, \mathrm{MeOD} / \mathrm{CDCl}_{3}\right.$ 1:1) E2 $\delta-5.57$. IR v 509; 721; 934; 976; 1050; 1082; 1165; 1238; 1267; 1467; 1755; 2849; 2916; 2956; 3389. HR-MS (ESI+) m/z $[\mathrm{M}-\mathrm{Cl}]^{+}$calcd for $\mathrm{C}_{35} \mathrm{H}_{73} \mathrm{NO}_{6} \mathrm{P}^{+}$634.5170, found 634.5167. Note: IR and HR-MS were measured on the mixture of the four diastereomers.

\section{4-(((Dodecyloxy)(octadecyloxy)phosphoryl)oxy)-1,1-dimethylpiperidin-1-ium chloride $\left(\boldsymbol{P}_{\mathrm{a}}\right)$}

Compound $\mathbf{P}_{\mathbf{a}}$ (55 mg, $48 \%$ ) was obtained from perifosine (79 $\mathrm{mg}, 0.17 \mathrm{mmol}$ ) and dodecyl triflate $(141 \mathrm{mg}, 0.44 \mathrm{mmol})$ according to the general procedure except the reaction was conducted at rt. $R_{\mathrm{f}}=0.68\left(\mathrm{CH}_{2} \mathrm{Cl}_{2} / \mathrm{MeOH} / \mathrm{H}_{2} \mathrm{O}\right.$ 75:22:3). ${ }^{1} \mathrm{H}-\mathrm{NMR}\left(400 \mathrm{MHz}, \mathrm{MeOD} / \mathrm{CDCl}_{3}\right.$ 1:1) $\delta 0.86(\mathrm{t}, J=6.7 \mathrm{~Hz}, 6 \mathrm{H}) ; 1.24(\mathrm{~m}, 48 \mathrm{H}) ; 1.68\left(\mathrm{tt}, J_{1}=J_{2}=7.0 \mathrm{~Hz}, 4 \mathrm{H}\right) ; 2.10-2.32(\mathrm{~m}$, $4 \mathrm{H}) ; 3.14(\mathrm{~s}, 3 \mathrm{H}) ; 3.20(\mathrm{~s}, 3 \mathrm{H}) ; 3.41-3.55(\mathrm{~m}, 4 \mathrm{H}) ; 4.06(\mathrm{~m}, 4 \mathrm{H}) ; 4.62(\mathrm{~m}, 1 \mathrm{H}) .{ }^{13} \mathrm{C}-\mathrm{NMR}$ $\left(100.7 \mathrm{MHz}, \mathrm{CDCl}_{3}\right) \delta 14.4$ (2C); 23.0 (2C); 25.7 (2C); 26.9 (2C); 29.6 (2C); 29.7 (2C); 29.9 (2C); 30.1 (5C); 30.2 (6C); 30.5; 30.6 (2C); 32.2 (2C); 47.1 (2C); 59.1 (2C); 69.2; 69.3 (2C). ${ }^{31} \mathrm{P}-\mathrm{NMR}\left(162 \mathrm{MHz} ; \mathrm{MeOD} / \mathrm{CDCl}_{3} 1: 1\right) \delta-2.07$. IR v 516; 573; 638; 721; 764; 923; 1029; $1161 ; 1245 ; 1467 ; 2851 ; 2920 ; 3252$. HR-MS (ESI +$) \mathrm{m} / \mathrm{z}[\mathrm{M}-\mathrm{Cl}]^{+}$calcd for $\mathrm{C}_{37} \mathrm{H}_{77} \mathrm{NO}_{4} \mathrm{P}^{+}$ 630.5585 , found 630.5581 . 


\section{4-((((Dodecanoyloxy)methoxy)(octadecyloxy)phosphoryl)oxy)-1, 1-dimethylpiperidin-}

\section{1-ium chloride $\left(\boldsymbol{P}_{\boldsymbol{b}}\right)$}

Compound $\mathbf{P}_{\mathbf{b}}(51 \mathrm{mg}, 22 \%)$ was obtained from perifosine $(150 \mathrm{mg}, 0.33 \mathrm{mmol})$ and chloromethyl dodecanoate $(675 \mathrm{mg}, 2.70 \mathrm{mmol})$ according to the general procedure. $R_{\mathrm{f}}: 0.6$ $\left(\mathrm{CH}_{2} \mathrm{Cl}_{2} / \mathrm{MeOH} / \mathrm{H}_{2} \mathrm{O} 75: 22: 3\right) .{ }^{1} \mathrm{H}-\mathrm{NMR}\left(400 \mathrm{MHz}, \mathrm{MeOD} / \mathrm{CDCl}_{3} 1: 1\right) \delta 0.86(\mathrm{t}, J=6.7 \mathrm{~Hz}$, $6 \mathrm{H}) ; 1.24(\mathrm{~m}, 46 \mathrm{H}) ; 1.63\left(\mathrm{tt}, J_{1}=J_{2}=6.9 \mathrm{~Hz}, 2 \mathrm{H}\right) ; 1.68\left(\mathrm{tt}, J_{l}=J_{2}=7.2 \mathrm{~Hz}, 2 \mathrm{H}\right) ; 2.10-2.32$ $(\mathrm{m}, 4 \mathrm{H}) ; 2.39(\mathrm{t}, J=7.6 \mathrm{~Hz}, 2 \mathrm{H}) ; 3.17(\mathrm{~s}, 3 \mathrm{H}) ; 3.22(\mathrm{~s}, 3 \mathrm{H}) ; 3.45-3.59(\mathrm{~m}, 4 \mathrm{H}) ; 4.08(\mathrm{td}$, $\left.J_{l}=J_{2}=6.8 \mathrm{~Hz}, 2 \mathrm{H}\right) ; 4.70(\mathrm{~m}, 1 \mathrm{H}) ; 5.62 \quad\left(\mathrm{ABX}\right.$ syst., $J_{A B}=5.2 \mathrm{~Hz}, J_{A X}=13.8 \mathrm{~Hz}$, $\left.J_{B X}=11.1 \mathrm{~Hz}, 2 \mathrm{H}\right) .{ }^{13} \mathrm{C}-\mathrm{NMR}\left(100,7 \mathrm{MHz} ; \mathrm{MeOD} / \mathrm{CDCl}_{3} 1: 1\right) \delta 14.5$ (2C); 23.4 (2C); 25.3; $26.1 ; 27.1$ (2C); 29.8; 29.9; 30.0; 30.1 (2C); 30.2; 30.3 (5C); 30.4 (7C); 30.9; 32.6 (2C); 34.6; 54.7 (2C); 58.9 (2C); 69.9; 70.0; 83.5; 173.1. ${ }^{31} \mathrm{P}-\mathrm{NMR}\left(162 \mathrm{MHz}, \mathrm{MeOD} / \mathrm{CDCl}_{3} 1: 1\right) \delta-4.22$. IR $v 531 ; 666 ; 720 ; 760 ; 802 ; 923 ; 966 ; 1020 ; 1148 ; 1264 ; 1468 ; 1644 ; 1764 ; 2849 ; 2916$; 2955; 3378. HR-MS (ESI+) m/z [M-Cl] $]^{+}$calcd for $\mathrm{C}_{38} \mathrm{H}_{77} \mathrm{NO}_{6} \mathrm{P}^{+}$674.5483, found 674.5477.

\section{4-(((1-(Dodecanoyloxy)ethoxy)(octadecyloxy)phosphoryl)oxy)-1,1-dimethylpiperidin- 1-ium chloride $\left(\boldsymbol{P}_{c}\right)$}

Compound $\mathbf{P}_{\mathbf{c}}$ (96 mg, $20 \%$ ) was obtained as two separated couples of enantiomers (E1 and E2) from perifosine (300 mg, $0.65 \mathrm{mmol})$ and 1-chloroethyl dodecanoate (1.33 g, $5.06 \mathrm{mmol})$ according to the general procedure. $R_{\mathrm{f}}: 0.3(\mathrm{E} 1)$ and $0.4(\mathrm{E} 2)\left(\mathrm{CH}_{2} \mathrm{Cl}_{2} / \mathrm{MeOH} / \mathrm{H}_{2} \mathrm{O} 75: 22: 3\right)$. ${ }^{1} \mathrm{H}-\mathrm{NMR}\left(400 \mathrm{MHz}, \mathrm{MeOD} / \mathrm{CDCl}_{3}\right.$ 1:1) D1 $\delta 0.86(\mathrm{t}, J=6.7 \mathrm{~Hz}, 6 \mathrm{H}) ; 1.24(\mathrm{~m}, 46 \mathrm{H}) ; 1.56(\mathrm{~d}$, $J=5.2 \mathrm{~Hz}, 3 \mathrm{H}) ; 1.61\left(\mathrm{tt}, J_{1}=J_{2}=6.9 \mathrm{~Hz}, 2 \mathrm{H}\right) ; 1.68\left(\mathrm{tt}, J_{l}=J_{2}=7.2 \mathrm{~Hz}, 2 \mathrm{H}\right) ; 2.08-2.30(\mathrm{~m}$, 4H); $2.36(\mathrm{t}, J=7.6 \mathrm{~Hz}, 2 \mathrm{H}) ; 3.17(\mathrm{~s}, 3 \mathrm{H}) ; 3.24(\mathrm{~s}, 3 \mathrm{H}) ; 3.45-3.59(\mathrm{~m}, 4 \mathrm{H}) ; 4.06\left(\mathrm{td}, J_{I}=J_{2}=6.8 \mathrm{~Hz}\right.$, $2 \mathrm{H}) ; 4.70(\mathrm{~m}, 1 \mathrm{H}) ; 6.46\left(\mathrm{qd}, J_{l}=J_{2}=5.2 \mathrm{~Hz}, 1 \mathrm{H}\right) .{ }^{1} \mathrm{H}-\mathrm{NMR}\left(400 \mathrm{MHz}, \mathrm{MeOD} / \mathrm{CDCl}_{3} 1: 1\right) \mathrm{E} 2$ $\delta 0.86(\mathrm{t}, J=6.7 \mathrm{~Hz}, 6 \mathrm{H}) ; 1.24(\mathrm{~m}, 46 \mathrm{H}) ; 1.54(\mathrm{~d}, J=5.2 \mathrm{~Hz}, 3 \mathrm{H}) ; 1.61\left(\mathrm{tt}, J_{1}=J_{2}=6.9 \mathrm{~Hz}, 2 \mathrm{H}\right) ;$ $1.68\left(\mathrm{tt}, J_{I}=J_{2}=7.2 \mathrm{~Hz}, 2 \mathrm{H}\right) ; 2.08-2.30(\mathrm{~m}, 4 \mathrm{H}) ; 2.35(\mathrm{t}, J=7.6 \mathrm{~Hz}, 2 \mathrm{H}) ; 3.18(\mathrm{~s}, 3 \mathrm{H}) ; 3.24$ 
$(\mathrm{s}, 3 \mathrm{H}) ; 3.45-3.59(\mathrm{~m}, 4 \mathrm{H}) ; 4.08\left(\mathrm{td}, J_{l}=J_{2}=6.8 \mathrm{~Hz}, 2 \mathrm{H}\right) ; 4.70(\mathrm{~m}, 1 \mathrm{H}) ; 6.50 \quad(\mathrm{qd}$, $\left.J_{1}=J_{2}=5.2 \mathrm{~Hz}, 1 \mathrm{H}\right) .{ }^{13} \mathrm{C}-\mathrm{NMR}\left(100.7 \mathrm{MHz}, \mathrm{MeOD} / \mathrm{CDCl}_{3} 1: 1\right) \mathrm{E} 1 \delta 14.5$ (2C); 21.7; 23.4 (2C); $25.3 ; 26.1 ; 26.8 ; 27.1 ; 29.7 ; 29.8 ; 30.0 ; 30.1(2 \mathrm{C}) ; 30.2 ; 30.3(5 \mathrm{C}) ; 30.4(7 \mathrm{C}) ; 30.8 ; 32.6$ (2C); 34.7; 55.2 (2C); 58.6 (2C); 69.5; 69.7; 92.1; 173.1. ${ }^{13} \mathrm{C}-\mathrm{NMR} \quad(100.7 \mathrm{MHz}$, $\left.\mathrm{MeOD} / \mathrm{CDCl}_{3} 1: 1\right)$ E2 $\delta 14.5(2 \mathrm{C}) ; 21.7 ; 23.4(2 \mathrm{C}) ; 25.3 ; 26.1 ; 27.0 ; 27.1 ; 29.7 ; 29.8 ; 30.0$; 30.1 (2C); 30.2; 30.3 (5C); 30.4 (7C); 30.8; 32.6 (2C); 34.7; 54.8 (2C); 58.8 (2C); 69.7; 69.9; 92.3; 172.8. ${ }^{31} \mathrm{P}-\mathrm{NMR}\left(162 \mathrm{MHz}, \mathrm{MeOD} / \mathrm{CDCl}_{3} 1: 1\right)$ E1 $\delta-6.1 .{ }^{31} \mathrm{P}-\mathrm{NMR}(162 \mathrm{MHz}$, $\left.\mathrm{MeOD} / \mathrm{CDCl}_{3} 1: 1\right)$ E2 $\delta-6.0$. IR $v 553 ; 638 ; 772 ; 752 ; 974 ; 1012 ; 1090 ; 1163 ; 1263 ; 1466$; $1745 ; 2851 ; 2920 ; 3380$. HR-MS (ESI+) m/z $[\mathrm{M}-\mathrm{Cl}]^{+}$calcd for $\mathrm{C}_{39} \mathrm{H}_{79} \mathrm{NO}_{6} \mathrm{P}^{+}$688.5640, found 688,5627. Note: IR and HR-MS were measured on the mixture of the four diastereomers.

\section{RESULTS AND DISCUSSION}

\section{Design and Synthesis of the pro-APLs}

Phosphotriesters as those presented at figure 2 may be considered as potential APL prodrugs. Nevertheless, three different phosphodiesters will result from their non-selective hydrolysis, of which the expected APL. The two other phosphodiesters will be produced through the alternate leaving of the 1-dodecanol or choline substituent. In order to avoid these unwanted side reactions, the parent APL should be derivatized through the grafting of a labile substituent. Under a suitable chemical or enzyme stimulus, selective removal of the latter may be obtained. Thus, considering our previous work on membrane phospholipid-based phosphoacetals as labile cationic lipids for gene delivery $(24,25)$, we explored the possibility to develop our methodology for producing potent prodrugs of APLs (Fig. 3). To this end, a dodecyl chain was tethered to the phosphate group of APLs, directly or through an acetal moiety. It has been previously demonstrated that a methyl substituent on the acetal bridge improves hydrolytic susceptibility of the phosphotriester (25). Therefore, this modification was 


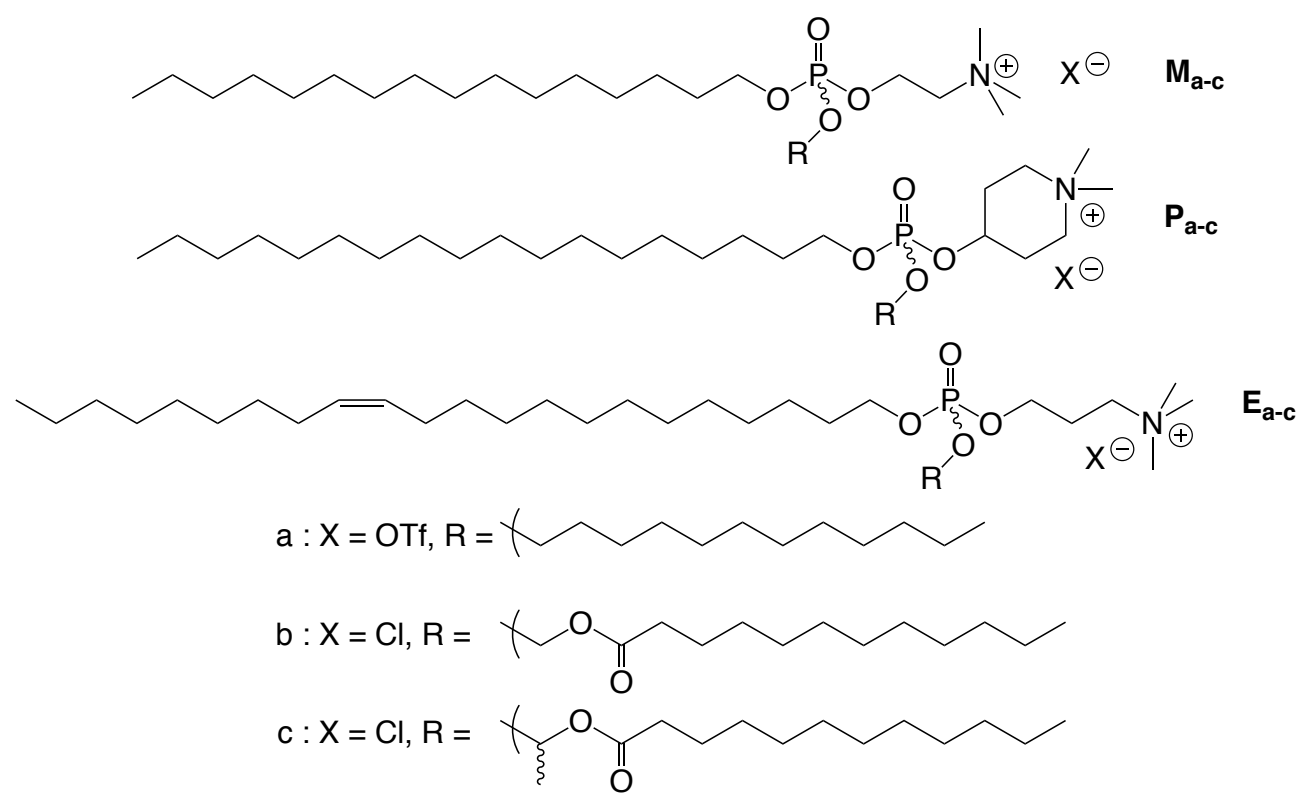

Figure 3 Structure of the pro-APL compounds described in this work.

introduced in the pro-APL series. As reference compounds and starting materials for the preparation of pro-APLs, miltefosine, perifosine and erufosine were first prepared (Sch. 1). Miltefosine was synthesized in two steps according to a protocol described by North et al. (28) with modifications allowing improvement of the reaction yield from 5.1 to $35 \%$. Perifosine was obtained using a one-pot procedure starting from 1-octadecanol, phosphorus oxychloride and $N, N$-dimethyl-4-hydroxypiperidinium tosylate. Erufosine was prepared in two

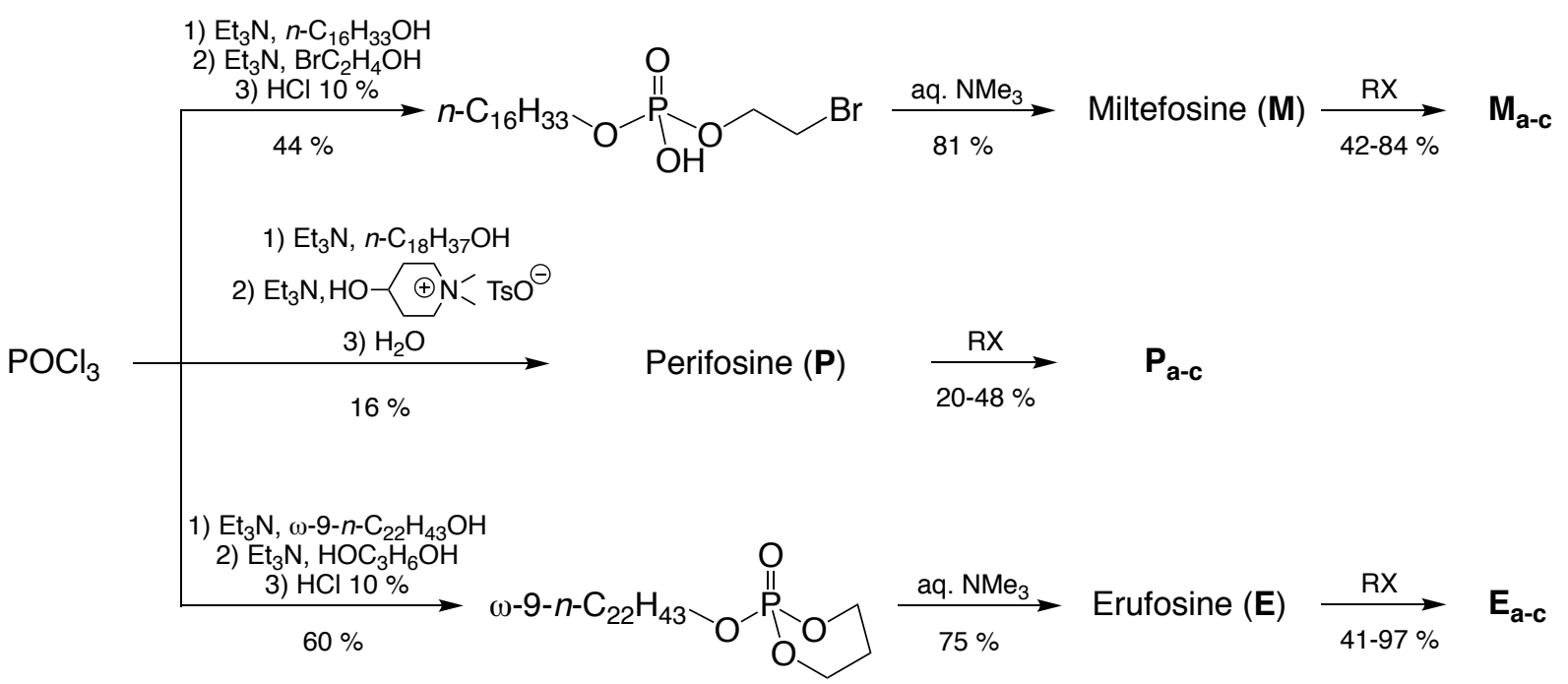

Scheme 1 Synthesis of the parent APLs, miltefosine, perifosine and erufosine, and subsequent pro-APLs. 
steps from erucyl alcohol, 1,3-propanediol, and phosphorus oxychloride (26). The intermediate 1,3-dioxa-2-phosphinane was reacted with trimethylamine to afford the target compound in $75 \%$ yield. The nine pro-APL compounds were then prepared through the direct $O$-alkylation

of APLs by adequate electrophilic reagents, namely 1-dodecyl triflate, chloromethyl dodecanoate, and 1-chloroethyl dodecanoate. Reaction yields were variable, depending on the nucleophilicity of the parent APL, perifosine offering lower reactivity presumably due to higher stability of the zwitterion. $\mathbf{M}_{\mathfrak{c}}, \mathbf{P}_{\mathbf{c}}$, and $\mathbf{E}_{\mathbf{c}}$ were obtained as two couples of enantiomers due to the two stereogenic centers in the molecule. In some cases, the isomers could be separated but evaluations (vide infra) were performed on the original mixture of diastereomers.

\section{Self-Assembly Properties of the pro-APLs}

There is broad consensus that APLs may act as detergent molecules and display membraneactive properties. At high concentration, these compounds impair the orderly structure of the plasma membrane. Micellar clusters are produced, leading to the formation of pores and holes in the membranes of tumor cells (29). In order to investigate the behavior of APLs and proAPLs in aqueous environment, the fluorescence probe technique was selected. This technique has been widely used to investigate micellization of surfactants and determine their CMC. In the case of amphiphilic compounds with a low hydrophilic-lipophilic balance (HLB) as it is most likely the case with the pro-APLs described herein, aggregation may preferentially proceed through the formation of lamellar structures. In this case, the concentration above which self-assembly occurs should be referred to the critical aggregation concentration (CAC) instead of CMC. In our study, was selected pyrene as the hydrophobic fluorescent dye. Pyrene fluorescence vary with the polarity of the solubilizing medium and various fluorescence patterns are observed where the dye environment is hydrophilic or lipophilic. Measurements were performed in water and data obtained for the APLs and pro-APLs are collected in Table I. 
Table I APLs and pro-APLs critical aggregation concentration as measured with pyrene by the fluorescent probe technique.

\begin{tabular}{lcccccccccccc}
\hline & $\mathbf{M}$ & $\mathbf{M}_{\mathbf{a}}$ & $\mathbf{M}_{\mathbf{b}}$ & $\mathbf{M}_{\mathbf{c}}$ & $\mathbf{P}$ & $\mathbf{P}_{\mathbf{a}}$ & $\mathbf{P}_{\mathbf{b}}$ & $\mathbf{P}_{\mathbf{c}}$ & $\mathbf{E}$ & $\mathbf{E}_{\mathbf{a}}$ & $\mathbf{E}_{\mathbf{b}}$ & $\mathbf{E}_{\mathbf{c}}$ \\
\hline $\mathrm{CAC}(\mu \mathrm{M})$ & 11.2 & 1.1 & 4.3 & 1.2 & 4.4 & 1.2 & 2.9 & 2.3 & 1.4 & 0.5 & 0.5 & 0.5 \\
\hline
\end{tabular}

The CMC values obtained for miltefosine, perifosine and erufosine were 11.2, 4.4, and 1.4 $\mu \mathrm{M}$, respectively. The $\mathrm{CMC}$ determined for miltefosine was consistent with that previously determined by the Du Noüy ring method $(12 \mu \mathrm{M})(30)$ whereas the Langmuir trough method yielded a somehow lower value $(2.5-3.0 \mu \mathrm{M})(31)$. For perifosine, the $\mathrm{CMC}$ was found to be $4.4 \mu \mathrm{M}$ which is similar to the value determined by the Langmuir trough method $(2.5 \mu \mathrm{M})$ (11). No CMC data for erufosine could be found in the literature. As expected, transformation of APLs into pro-APLs significantly decreased the CAC of the compounds. In the miltefosine series, the effect was appreciably greater with a ten-fold decrease in the CAC value when compared to the parent compound, whereas a two- to three-fold decrease was observed in the perifosine and erufosine series. This is explained by the longest alkyl chain residue of these two APLs and consequently, their strongest initial lipophilic nature.

\section{Hydrolytic Properties of the pro-APLs}

Aqueous formulation of pro-APLs results in the formation of nanosized lipid aggregates (liposomes) that most likely are internalized by cells through the endocytic route. To express their intrinsic antitumor activity, pro-APLs need to be transformed into APLs which are the active drugs. Such a transformation may may be triggered by a chemical stimulus, e.g., a pH decrease, such as the one observed in the endosomal compartment during the maturation process, or can be mediated by hydrolytic enzymes that are abundant in lysosomes. We thus investigated the resistance of the compounds to hydrolysis under neutral and acidic conditions, mimicking the extracellular and late endosome environment, respectively, using ${ }^{31} \mathrm{P}-\mathrm{NMR}$ 
spectroscopy. ${ }^{31} \mathrm{P}$ chemical shifts displayed by phosphotriesters and phosphodiesters differ by 5-6 ppm. This variation allows accurate monitoring of the hydrolysis reaction. The pro-APLs were processed into liposomes at neutral and acidic $\mathrm{pH}$, and incubated at $25^{\circ} \mathrm{C}$. Periodical acquisition of the ${ }^{31} \mathrm{P}-\mathrm{NMR}$ spectra under conditions enabling quantitative measurements (4sec pulse cycle) were carried out to determine the rate of hydrolysis (Fig. 4, Table II). As might be anticipated, phosphotriesters without acetal linker $\left(\mathbf{M}_{\mathbf{a}}, \mathbf{P}_{\mathbf{a}}\right.$, and $\left.\mathbf{E}_{\mathbf{a}}\right)$ retained their full structural integrity under neutral and acidic conditions, even over an extended period of time (>31d). Though to various extents, all the other compounds revealed sensitive to hydrolysis.
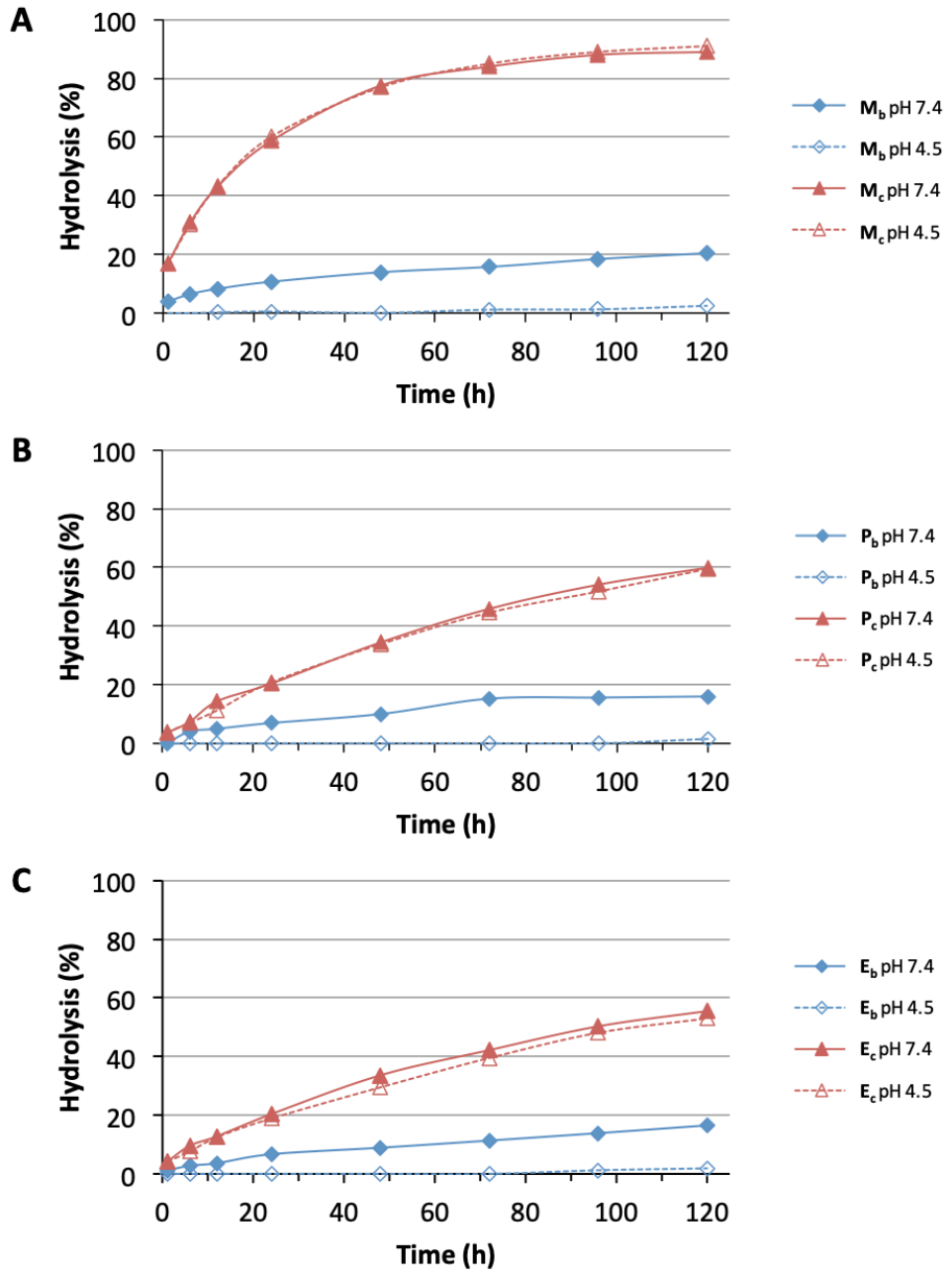

Figure 4 Hydrolytic stability of the pro-APLs. Compounds formulated into liposomes were incubated at $25^{\circ} \mathrm{C}$ and hydrolysis was monitored by quantitative ${ }^{31} \mathrm{P}-\mathrm{NMR}$ measurements, at $\mathrm{pH} 7.4$ and 4.5 (A: miltefosine prodrugs; $\mathrm{B}$ : perifosine prodrugs; C: erufosine prodrugs). 
Overall, hydrolysis of the compounds was accelerated at $\mathrm{pH} 7.4$ as compared to $\mathrm{pH} 4.5$. This indicates that mixed acetals of phosphoric and carboxylic esters do not display the same reactivity as dialkyl acetals which are fairly stable in neutral or basic media and highly labile under acidic conditions. These results are consistent with those previously obtained with other phosphoacetals $(24,25)$ and point out that hydrolysis of such compounds cannot be analyzed just considering the intrinsic reactivity of the acetal moiety but must be interpreted with regard to the respective sensitivity of the ester moieties (carboxylic and phosphoric) towards hydrolysis in aqueous solutions, depending on the reaction mechanism involved (specific base catalyzed, water catalyzed, or specific acid catalyzed). Fully consistent with prior examples in the literature, introduction of a methyl substituent on the acetal bridge also invariably provoked a drastic increase in the hydrolysis rate of the pro-APLs, both under basic and acidic conditions. In the miltefosine series, whereas only 3-20\% hydrolysis were measured after 5 days incubation of the compound lacking acetal substitution $\left(\mathbf{M}_{\mathbf{b}}\right)$, methyl substitution $\left(\mathbf{M}_{\mathbf{c}}\right)$ led to $50 \%$ hydrolysis within $17-18 \mathrm{~h}$, regardless of the $\mathrm{pH}$. This roughly corresponded to an acceleration of the hydrolysis rate by a factor of 5 at $\mathrm{pH} 7.4$, and by a factor of 120 at $\mathrm{pH} 4.5$. The same trend was observed in the perifosine and erufosine series.

Table II Susceptibility of the pro-APLs to hydrolysis. Pro-APLs were incubated at $\mathrm{pH} 7.4$ and 4.5, at $25^{\circ} \mathrm{C}$. Extent of the hydrolysis reaction was determined by quantitative ${ }^{31} \mathrm{P}-\mathrm{NMR}$ spectroscopy. $\mathrm{H}_{120}$ refers to the extent of hydrolysis (\%) after a 120-h incubation period. When possible, time required for $50 \%$ hydrolysis $\left(t_{1 / 2}\right)$ was calculated from the theoretical curve fitting with the experimental data.

\begin{tabular}{lcccccccccc}
\hline & & $\mathbf{M}_{\mathbf{a}}{ }^{*}$ & $\mathbf{M}_{\mathbf{b}}$ & $\mathbf{M}_{\mathbf{c}}$ & $\mathbf{P}_{\mathbf{a}}{ }^{*}$ & $\mathbf{P}_{\mathbf{b}}$ & $\mathbf{P}_{\mathbf{c}}$ & $\mathbf{E}_{\mathbf{a}}{ }^{*}$ & $\mathbf{E}_{\mathbf{b}}$ & $\mathbf{E}_{\mathbf{c}}$ \\
\hline $\mathrm{H}_{120}(\%)$ & $\mathrm{pH} \mathrm{7.4}$ & 0 & 20 & 89 & 0 & 16 & 60 & 0 & 17 & 56 \\
& $\mathrm{pH} \mathrm{4.5}$ & 0 & 3 & 91 & 0 & 2 & 60 & 0 & 2 & 53 \\
\hline \multirow{2}{*}{$t_{1 / 2}(\mathrm{~h})$} & $\mathrm{pH} \mathrm{7.4}$ & - & - & 18 & - & - & 84 & - & - & 96 \\
& $\mathrm{pH} \mathrm{4.5}$ & - & - & 17 & - & - & 90 & - & - & 104 \\
\hline
\end{tabular}

No hydrolysis was observed after an incubation period of $31 \mathrm{~d}$. 


\section{Hemolytic Activity of the pro-APLs}

Most APLs described to date display hemolytic activity that is incompatible with administration through the iv route $(7,13)$. Though hemolysis can be partly prevented if the APLs are processed in liposomal form (i.e., co-formulation with other lipids such as cholesterol and 1,2-dipalmitoyl-sn-glycero-phosphoglycerol) $(32,33)$, a significant reduction in therapeutic activity is achieved in this case, as previously shown in vitro (34). The presence of micelles is important for the cell lysis mechanism, which involves micellar dissolution and partition into the cell membrane, phase transition between lamellae and micelles and decrease in the size of micelles (35). In the absence of serum, it has been shown that the lytic concentration for different APLs and related compounds correspond rather well to their CMC which are in the low $\mu \mathrm{M}$ range $(7,36)$. Thus, lowering the CMC of an APL through the formation of a more hydrophobic prodrug, a pro-APL, might result in improving the biocompatibility of the compound. In order to examine this hypothesis, the hemolytic activity of APLs and pro-APLs was measured. Sheep red blood cells (RBC) were incubated at $37^{\circ} \mathrm{C}$

A

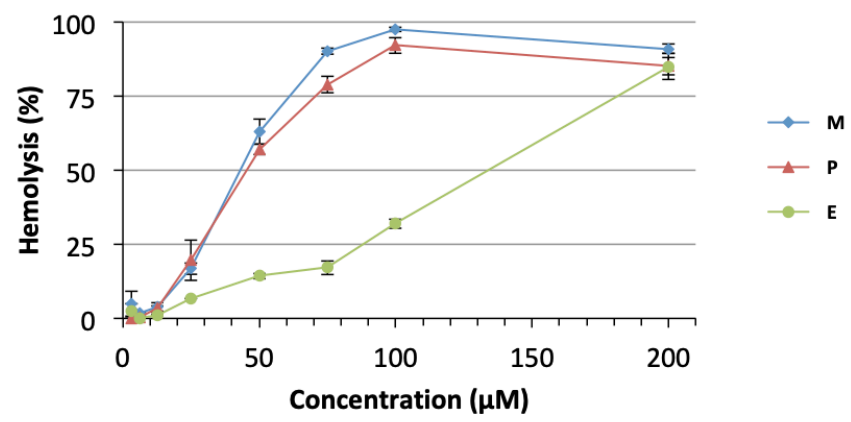

B

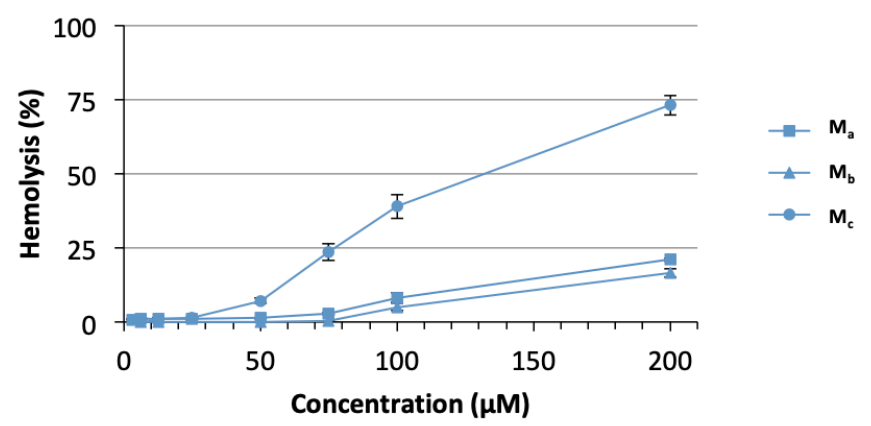

Figure 5 Hemolytic activity of APLs (A) and miltefosine prodrugs (B) incubated with sheep RBC at $37^{\circ} \mathrm{C}$ for $1 \mathrm{~h}$. 
with increasing doses of the compounds and hemolysis was monitored spectrophotometrically. As expected, hemolysis increased in a concentration-dependent manner (Fig. 5). However, whereas APLs provoked massive hemolysis within $1 \mathrm{~h}$ (Fig. 5A),pro-APLs revealed much less deleterious to RBC. At the highest concentration tested $(200 \mu \mathrm{M}), \mathbf{M}_{\mathbf{a}}, \mathbf{M}_{\mathbf{b}}$, and $\mathbf{M}_{\mathbf{c}}$ only provoked $21 \%, 16 \%$, and $73 \%$ hemolysis, respectively (Fig. 5B). By comparison, miltefosine provoked complete disruption of $\mathrm{RBC}$ at the concentration of $c a .100 \mu \mathrm{M}$. The higher hemolytic toxicity of $\mathbf{M}_{\mathbf{c}}$, as compared to $\mathbf{M}_{\mathbf{a}}$ and $\mathbf{M}_{\mathbf{b}}$, was consistent with the lower hydrolytic stability of this compound (vide supra), hemolysis being caused by miltefosine that was produced in situ. All the other pro-APLs investigated herein did not significantly alter RBC (hemolysis $<2 \%$ ) over a 1-h incubation period. Thus, in order to characterize and discriminate the pro-APL compounds, incubation time was extended and hemolysis was measured at $24 \mathrm{~h}$ (Fig. 6). Concentration of the compounds inducing $50 \%$ hemolysis (hemolytic concentration for $50 \%$ effect, $\mathrm{HC}_{50}$ ) and percentage of hemolysis induced at the concentration of $200 \mu \mathrm{M}$ (hemolytic activity at $200 \mu \mathrm{M}, \mathrm{HA}_{200}$ ) are displayed in Table III. Among the three pro-APLs series, miltefosine prodrugs displayed the higher residual hemolytic toxicity, whereas proerufosine compounds had only minimal effect on $\mathrm{RBC}$, and perifosine prodrugs exhibited intermediate properties. Besides, as might be anticipated, the more labile the compounds, the higher the hemolysis, and $\mathbf{M}_{\mathbf{c}}, \mathbf{P}_{\mathbf{c}}$, and $\mathbf{E}_{\mathbf{c}}$ were invariably associated with the higher residual hemolytic toxicity in each prodrug series. Nevertheless, the data clearly show that pro-APLs display drastically reduced hemolytic effect as compared to the parent APLs. Hemolysis provoked by perifosine and miltefosine prodrugs after $24 \mathrm{~h}$ was much lower in most cases than that induced by erufosine itself after only $1 \mathrm{~h}$. Besides, erufosine prodrugs were practically devoided of any hemolytic activity $\left(\mathrm{HA}_{200}<25 \%\right.$, after $\left.24 \mathrm{~h}\right)$. As erufosine is the only APL currently considered for intravenous administration (37), it can be concluded that all the proAPLs described herein could be safely qualified for this administration route. 

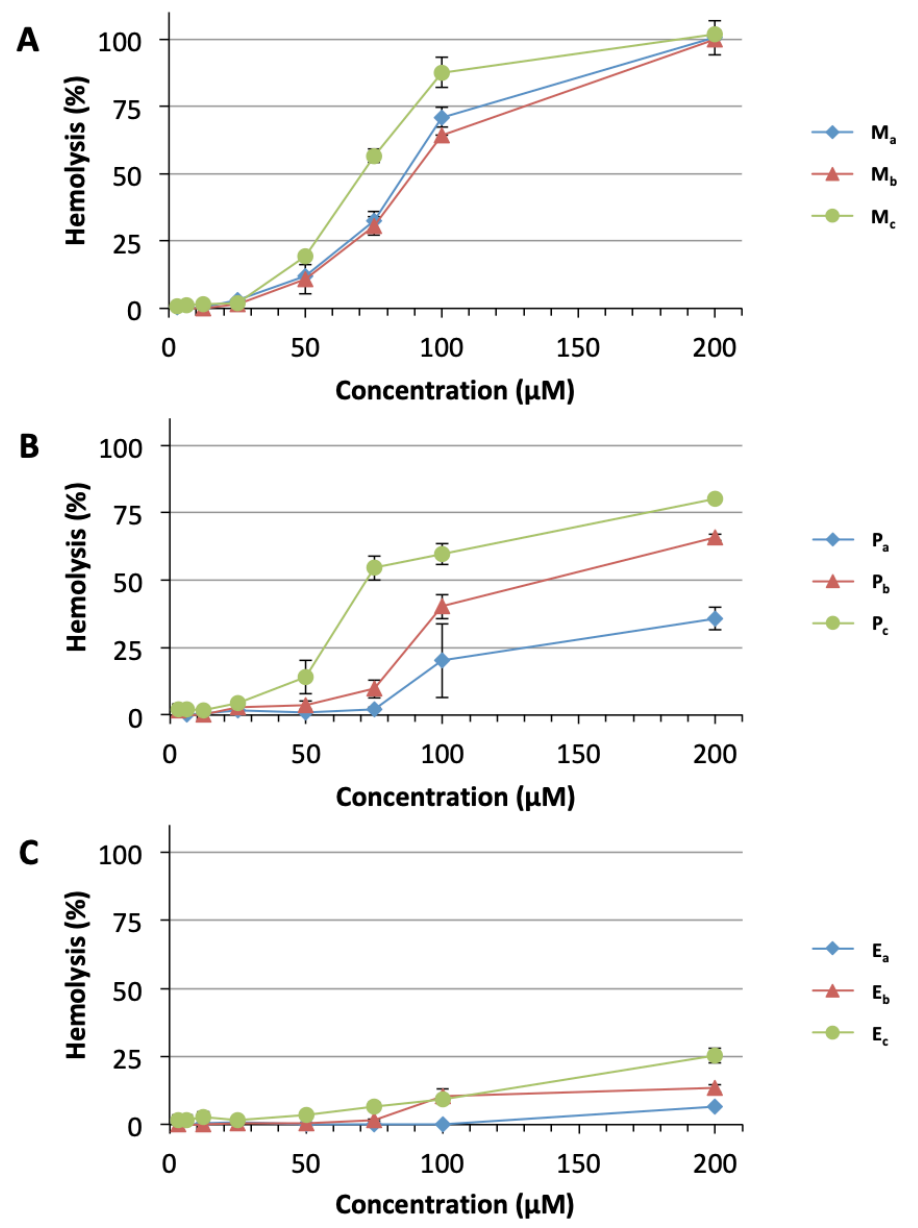

Figure 6 Hemolytic properties of the pro-APLs incubated with sheep red blood cells at $37^{\circ} \mathrm{C}$ for $24 \mathrm{~h}$.

Table III Hemolytic activity of pro-APLs upon incubation with sheep RBC for $24 \mathrm{~h}$ at $37^{\circ} \mathrm{C}$.

\begin{tabular}{lccccccccc}
\hline & $\mathbf{M}_{\mathrm{a}}$ & $\mathbf{M}_{\mathbf{b}}$ & $\mathbf{M}_{\mathbf{c}}$ & $\mathbf{P}_{\mathrm{a}}$ & $\mathbf{P}_{\mathbf{b}}$ & $\mathbf{P}_{\mathbf{c}}$ & $\mathbf{E}_{\mathrm{a}}$ & $\mathbf{E}_{\mathrm{b}}$ & $\mathbf{E}_{\mathrm{c}}$ \\
\hline $\mathrm{HC}_{50}(\mu \mathrm{M})^{\mathrm{a}}$ & 84 & 89 & 69 & $>200$ & 141 & 84 & $>200$ & $>200$ & $>200$ \\
$\mathrm{HA}_{200}(\%)^{\mathrm{b}}$ & 100 & 100 & 100 & 36 & 21 & 80 & 12 & 19 & 25
\end{tabular}

${ }^{\mathrm{a}}$ Concentration of pro-APL inducing $50 \%$ hemolysis. ${ }^{\mathrm{b}}$ Percentage of hemolysis provoked by the compound at $200 \mu \mathrm{mol}$.

\section{Cytotoxic Activity of the pro-APLs}

In the presence of serum, direct membrane permeabilization and cell lysis is not a major cytotoxic mechanism of action of APLs at pharmacologically relevant concentration $(38,39)$. It is widely appreciated that, at clinical dose, APLs insert into the cell membrane, producing a biophysical 
disturbance by altering cholesterol homeostasis, and finally disturbing various signal transduction pathways (40). To evaluate the antiproliferative effect of the APL prodrugs, a concentrationresponse study was performed in tumor and non-tumor pulmonary cells: A549 (human epithelial lung carcinoma), NCI-H292 (mucoepidermoid pulmonary carcinoma), and 16HBE (bronchial epithelial cells). The nine pro-APLs were assayed and compared with parent miltefosine, perifosine, and erufosine. Cells were exposed to concentration of lipids between $500 \mathrm{nM}$ and $1 \mathrm{mM}$, and cell survival was determined after a 24-h incubation period by the MTT dye reduction assay. A decrease in cell viability was observed in response to increasing concentration of APLs and pro-APLs in the three cell lines (Fig. 7). However, the cell lines showed different sensitivity to micellar APLs and lamellar pro-APLs, possibly indicating various mechanisms for the binding or uptake. The observation that no complete suppression of cell viability was attained, even at the highest concentration of $1 \mathrm{mM}$, is related to the known partial underestimation of antiproliferative agents by the MTT assay (41). The $\mathrm{IC}_{50}$ values (concentration that inhibited the cell growth by $50 \%$ ) reflecting the cytotoxicity of the compounds interpolated from the experimental concentration-effect curves are presented in Table IV. The $\mathrm{IC}_{50}$ values were in the range 39-192 $\mu \mathrm{M}$ for A549 cells, 46-168 $\mu \mathrm{M}$ for NCI-H292 cells, and 19-231 $\mu \mathrm{M}$ for non-cancerous $16 \mathrm{HBE}$ cells. The cytotoxicity of pro-APLs did not correlate with their previously measured hydrolytic stability. This likely originated from in situ enzyme-mediated hydrolysis of the pro-APL compounds, a process competing with pure $\mathrm{pH}$-controlled chemical hydrolysis. Depending on the pro-APL (i.e., the structure of the parent APL and that of the substituent grafted to the phosphoryl moiety), the compound may be metabolized into the the parent cytotoxic APL at various rates by lipases in the milieu. Anyhow, every pro-APL tested herein displayed a cytotoxicity that compared to that of the parent APL, revealing that it was productively metabolized into the cells. Nevertheless, depending on the cell line investigated, some pro-APLs revealed in some cases more active than the corresponding APL 

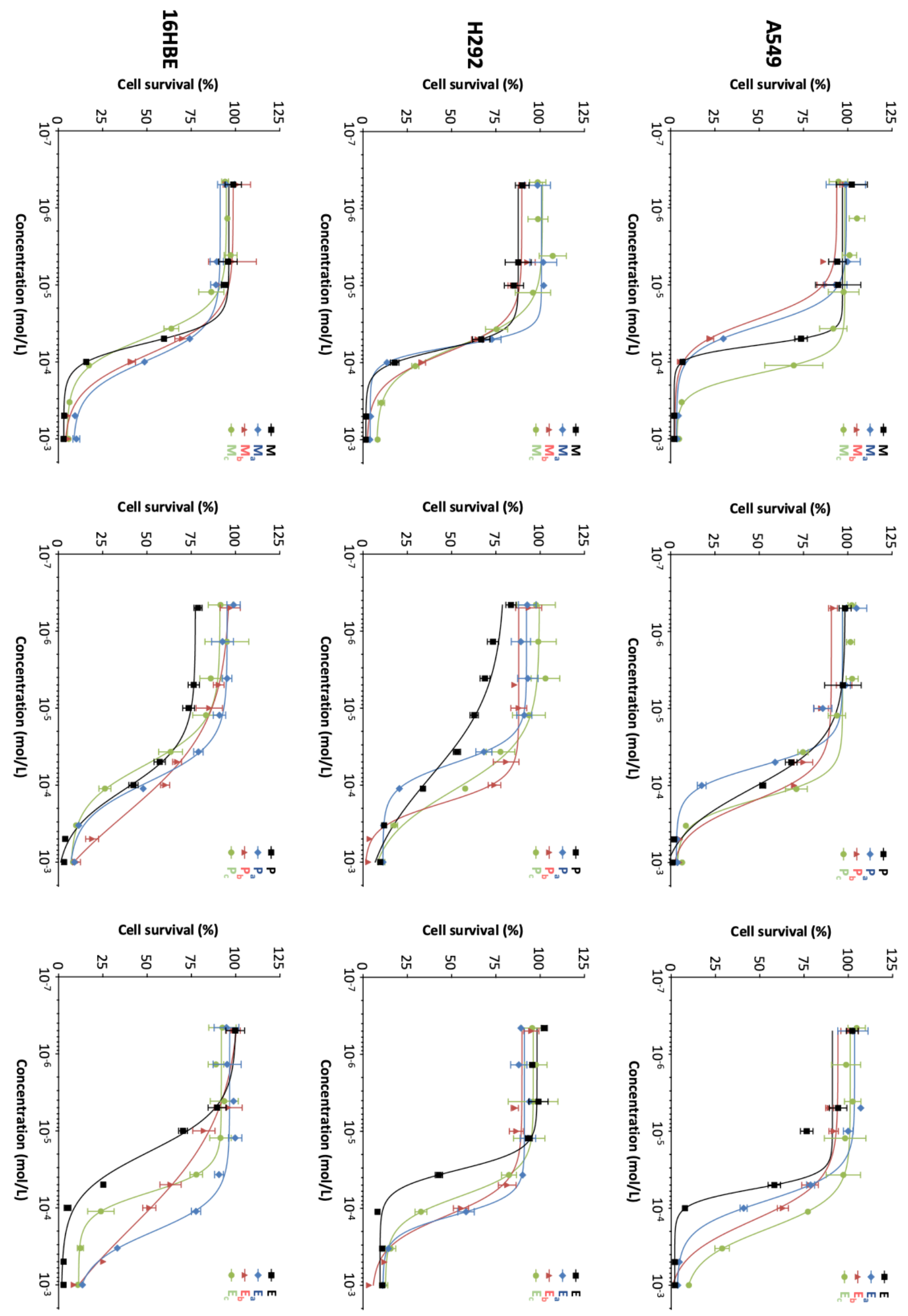

Figure 7. Survival of $\mathrm{A} 549, \mathrm{NCl}-\mathrm{H} 292$, and $16 \mathrm{HBE}$ cells treated with increasing concentrations of APLs and pro-APLs. 
Table IV Cytotoxic effect $\left(\mathrm{IC}_{50}\right)$ of APLs and pro-APLs in three cell lines exposed for $24 \mathrm{~h}$ at $37^{\circ} \mathrm{C}$, as measured by the MTT assay.

\begin{tabular}{lccc}
\hline & & $\mathrm{IC}_{50}(\mu \mathrm{M})$ & \\
\cline { 2 - 4 } Compound & $\mathrm{A} 549$ & $\mathrm{NCl}-\mathrm{H} 292$ & $16 \mathrm{HBE}$ \\
\hline $\mathbf{M}$ & $77 \pm 15$ & $70 \pm 9$ & $73 \pm 8$ \\
$\mathbf{M}_{\mathbf{a}}$ & $46 \pm 11$ & $56 \pm 7$ & $107 \pm 14$ \\
$\mathbf{M}_{\mathbf{b}}$ & $39 \pm 16$ & $52 \pm 28$ & $69 \pm 26$ \\
$\mathbf{M}_{\mathbf{c}}$ & $139 \pm 32$ & $59 \pm 10$ & $49 \pm 6$ \\
$\mathbf{P}$ & $127 \pm 39$ & $77 \pm 3$ & $116 \pm 33$ \\
$\mathbf{P}_{\mathbf{a}}$ & $56 \pm 7$ & $63 \pm 5$ & $72 \pm 7$ \\
$\mathbf{P}_{\mathbf{b}}$ & $139 \pm 4$ & $168 \pm 7$ & $231 \pm 70$ \\
$\mathbf{P}_{\mathbf{c}}$ & $148 \pm 38$ & $116 \pm 25$ & $60 \pm 5$ \\
$\mathbf{E}$ & $57 \pm 8$ & $46 \pm 8$ & $19 \pm 3$ \\
$\mathbf{E}_{\mathbf{a}}$ & $81 \pm 7$ & $124 \pm 11$ & $225 \pm 31$ \\
$\mathbf{E}_{\mathbf{b}}$ & $151 \pm 42$ & $125 \pm 26$ & $144 \pm 66$ \\
$\mathbf{E}_{\mathbf{c}}$ & $192 \pm 44$ & $73 \pm 15$ & $63 \pm 7$ \\
\hline
\end{tabular}

(see e.g. miltefosine and perifosine prodrugs in A549 and NCI-H292 cells). One explanation would be a higher internalization rate through the endocytic uptake routes and/or a more efficient addressing of the pro-APL or its hydrolysis product to the plasma membrane. In the erufosine series, such an effect was not observed and none of the prodrugs offered enhanced cytotoxicity when compared to the APL.

\section{CONCLUSION}

In the current study, we investigated biolabile prodrugs of APLs for the first time. The prodrugs were obtained through direct $O$-alkylation of miltefosine, perifosine, and erufosine with various electrophilic reagents (dodecyl triflate, chloromethyl dodecanoate, and 1- 
chloroethyl dodecanoate). Aggregation properties of the resulting pro-APLs were examined and their critical aggregation concentration was determined. Transformation of APLs into pro-APLs resulted in a significant decrease in water solubility of the compounds and, consequently, in their CAC. Besides, the hydrolytic stability of the pro-APLs was determined under neutral and acidic conditions mimicking the extracellular environment and the late endosome milieu, respectively. Pro-APLs incorporating a methyl substituted phosphoacetal bridge revealed exacerbated sensitivity to hydrolysis, both under neutral and acidic conditions. Furthermore, the decrease in CAC for the pro-APLs, as compared to the parent APLs, did translate into a drastic reduction of the hemolytic toxicity of the compounds. All the pro-APLs were less harmful to red blood cells than erufosine, the only APL that has been considered for intravenous administration to date. In order to determine whether pro-APLs inherited the antineoplastic activity of the parent APLs, three different cell lines were exposed to increasing concentrations of the compounds and $\mathrm{IC}_{50}$ were determined. Cytotoxicity of the pro-APLs was found in the low micromolar range, similar to that of APLs and thus revealing that the APL prodrugs were productively metabolized by cells into the active parent compounds. In conclusion, our results indicate that pro-APLs are intravenously injectable derivatives of APLs with similar antiproliferative efficacy. Their use could thus address one of the major issues met in cancer therapies involving antitumor lipids and restricting their utilization to oral and topical administration, because of limited maximum tolerated dose. Therefore pro-APLs may be suggested as an interesting therapeutic option, not only for cancer, but also for the treatment of other diseases (e.g., visceral and cutaneous leishmaniasis).

\section{ACKNOWLEDGEMENTS}

The authors are grateful for financial support to BG from Labex Medalis, Région Alsace, and Alsace contre le cancer. 


\section{CONFLICTS OF INTEREST}

The authors have no competing interests to declare.

\section{SUPPLEMENTARY DATA}

The detailed description of the materials and methods used to characterize the hydrolytic stability, self-assembly properties, hemolytic activity, and cytotoxicity of the compounds as well as the ${ }^{1} \mathrm{H}-,{ }^{13} \mathrm{C}$-, and ${ }^{31} \mathrm{P}-\mathrm{NMR}$ spectra for compounds $\mathbf{M}_{\mathbf{a}}, \mathbf{M}_{\mathbf{b}}, \mathbf{M}_{\mathbf{c}}, \mathbf{P}_{\mathbf{a}}, \mathbf{P}_{\mathbf{b}}$, and $\mathbf{P}_{\mathbf{c}}$ are provided as Supplementary Information and can be found at http://...

\section{REFERENCES}

1. Modolell M, Andreesen R, Pahlke W, Brugger U, Munder PG. Disturbance of phospholipid metabolism during the selective destruction of tumor cells induced by alkyl lysophospholipids. Cancer Res. 1979;39(11):4681-4686.

2. Kostadinova A, Topouzova-Hristova T, Momchilova A, Tzoneva R, Berger MR. Antitumor lipids - Structure, functions, and medical applications. Adv Protein Chem Struct Biol. 2015;101:27-66.

3. Breiser A, Kim DJ, Fleer EAM, Damenz W, drube A, Berger M, Nagel GA, Eibl H, Unger C. Distribution and metabolism of hexadecylphosphocholine in mice. Lipids. $1987 ; 22: 925-926$.

4. Yanapirut $P$, Berger MR, Reinhardt $M$, Schmähl D. In vitro investigations on the antineoplastic effect of hexadecylphosphocholine. Arzneimittelforschung. 1991;41:652655.

5. Eibl H, Hilgard P, Stekar J, Voegeli R, Harleman JH. Experimental therapeutic studies with miltefosine in rats and mice. Prog Exp Tumor Res. 1992;34:116-130. 
6. Verweij J, Planting AST, Stoter G, Gandia D, Armand JP. Phase II study of oral miltefosine in patients with squamous cell head and neck cancer. Eur J Cancer. 1993;29:778-779.

7. Kötting J, Marschner NW, Neumuller W, Unger C, Eibl H. Hexadecylphosphocholine and octadecyl-methyl-glycero-3-phosphocholine: a comparison of hemolytic activity, serum binding and tissue distribution. Prog Exp Tumor Res. 1992;34:131-142.

8. Hilgard P, Klenner T, Stekar J, Nössner G, Kutscher B, Engel J. D-21266, a new heterocyclic alkylphospholipid with antitumour activity. Eur J Cancer. 1997;33:442-446.

9. Gills JJ, Dennis PA. Perifosine: update on a novel Akt inhibitor. Curr Oncol Rep. 2009;11:102-110.

10. Vink SR, Schellens JHM, van Blitterswijk WJ, Verheij M. Tumor and normal tissue pharmacokinetics of perifosine, an oral anti-cancer alkylphospholipid. Invest New Drugs. $2005 ; 23: 279-286$.

11. Mravljak J, Reiner Z, Pečar S. Synthesis and biological evaluation of spin-labeled alkylphospholipid analogs. J Med Chem. 2005;48:6393-6399.

12. Hideshima T, Catley L, Yasui, Hiroshi, Ishitsuka K, Raje N, Mitsiades C, Podar K, Munshi NC, Chauhan D, Richardson PG, Anderson KC. Perifosine, an oral bioactive novel alkylphospholipid, inhibits Akt and induces in vitro and in vivo cytotoxicity in human multiple myeloma cells. Blood. 2006;107:4053-4062.

13. Ernst DS, Eisenhauer E, Wainman N, Davis M, Lohmann R, Baetz T, Belanger K, Smylie M. Phase II study of perifosine in previously untreated patients with metastatic melanoma. Invest New Drugs. 2005;23:569-575.

14. Leighl NB, Dent S, Clemons M, Vandenberg TA, Tozer R, Warr DG, Crump RM, Hedley D, Pond GR, Dancey JE, Moore MJ. A Phase II study of perifosine in advanced or metastatic breast cancer. Breast Cancer Res Treat. 2008;108:87-92. 
15. Cirstea D, Hideshima T, Rodig S, Santo L, Pozzi S, Vallet S, Ikeda H, Perrone G, Gorgun G, Patel K, Desai N, Sportelli P, Kapoor S, Vali S, Mukherjee S, Munshi NC, Anderson KC, Raje N. Dual inhibition of Akt/mammalian target of rapamycin pathway by nanoparticle albumin-bound-rapamycin and perifosine induces antitumor activity in multiple myeloma. Mol Cancer Ther. 2010;9:963-975.

16. Kötting J, Berger MR, Unger C, Eibl H. Alkylphosphocholines: influence of structural variation on biodistribution at antineoplastically active concentrations. Cancer Chemother Pharmacol. 1992;30:105-112.

17. Eibl H, Kaufmann-Kolle P. Medical application of synthetic phospholipids as liposomes and drugs. J Liposome Res. 1995;5:131-148.

18. Yosifov DY, Todorov PT, Zaharieva MM, Georgiev KD, Pilicheva BA, Konstantinov SM, Berger MR. Erucylphospho-N,N,N-trimethylpropylammonium (erufosine) is a potential antimyeloma drug devoid of myelotoxicity. Cancer Chemother Pharmacol. $2011 ; 67: 13-25$.

19. Bagley RG, Kurtzberg L, Rouleau C, Yao M, Teicher BA. Erufosine, an alkylphosphocholine, with differential toxicity to human cancer cells and bone marrow cells. Cancer Chemother Pharmacol. 2011;68:1537-1546.

20. Ríos-Marco P, Marco C, Gálvez X, Jiménez-López JM, Carrasco MP. Alkylphospholipids: an update on molecular mechanisms and clinical relevance. Biochim Biophys Acta-Biomembr. 2017;1859:1657-1667.

21. Wang L, Koynova R, Parikh H, MacDonald RC. Transfection activity of binary mixtures of cationic O-substituted phosphatidylcholine derivatives: The hydrophobic core strongly modulates physical properties and DNA delivery efficacy. Biophys J. 2006;91:36923706. 
22. Opstad CL, Zeeshan M, Zaidi A, Sliwka H-R, Partali V, Nicholson DG, Surve C, Izower MA, Bilchuk N, Lou HH, Leopold PL, Larsen H, Liberska A, Khalique NA, Raju L, Flinterman M, Jubeli E, Pungente MD. Novel cationic polyene glycol phospholipids as DNA transfer reagents-Lack of a structure-activity relationship due to uncontrolled selfassembling processes. Chem Phys Lipids. 2014;183:117-136.

23. Pierrat P, Creusat G, Laverny G, Pons F, Zuber G, Lebeau L. A cationic phospholipiddetergent conjugate as a new efficient carrier for siRNA delivery. Chem-Eur $\mathrm{J}$. $2012 ; 18: 3835-3839$.

24. Pierrat P, Kereselidze D, Wehrung P, Zuber G, Pons F, Lebeau L. Bioresponsive deciduous-charge amphiphiles for liposomal delivery of DNA and siRNA. Pharm Res. 2013;30:1362-1379.

25. Pierrat P, Laverny G, Creusat G, Wehrung P, Strub J-M, VanDorsselaer A, Pons F, Zuber G, Lebeau L. Phospholipid-detergent conjugates as novel tools for siRNA delivery. Chem-Eur J. 2013;19:2344-2355.

26. Gaillard B, Remy J-S, Pons F, Lebeau L. Cationic erufosine (ErPC3) prodrugs as gene delivery reagents for antitumor combined therapy. Chem-Eur J. 2019;25:15662-15679.

27. Heyes JA, Niculescu-Duvaz D, Cooper RG, Springer CJ. Synthesis of novel cationic lipids: effect of structural modification on the efficiency of gene transfer. J Med Chem. 2002;45:99-114.

28. North EJ, Osborne DA, Bridson PK, Baker DL, Parrill AL. Autotaxin structure-activity relationships revealed through lysophosphatidylcholine analogs. Bioorg Med Chem. $2009 ; 17: 3433-3442$.

29. Noseda A, White JG, Godwin PL, Jerome WG, Modest EJ. Membrane damage in leukemic cells induced by ether and ester lipids - An electron microscopic study. Exp Mol Pathol. 1989;50:69-83. 
30. Yaseen M, Wang Y, Su TJ, Lu JR. Surface adsorption of zwitterionic surfactants: n-alkyl phosphocholines characterised by surface tensiometry and neutron reflection. J Colloid Interface Sci. 2005;288:361-370.

31. Rakotomanga M, Loiseau PM, Saint-Pierre-Chazalet M. Hexadecylphosphocholine interaction with lipid monolayers. Biochim Biophys Acta-Biomembr. 2004;1661:212218.

32. Fichtner I, Zeisig R, Naundorf H, Jungmann S, Arndt D, Asongwe G, Double JA, Bibby MC. Antineoplastic activity of allkylphosphocholines (APC) in human breast carcinomas in vivo and in vitro - Use of liposomes. Breast Cancer Res Treat. 1994;32:269-279.

33. Kaufmann-Kolle P, Drevs J, Berger MR, Kotting J, Marschner N, Unger C, Eibl H. Pharmacokinetic behavior and antineoplastic activity of liposomal hexadecylphosphocholine. Cancer Chemother Pharmacol. 1994;34:393-398.

34. Zeisig R, Jungmann S, Arndt D, Schutt A, Nissen E. Antineoplastic activity in vitro of free and liposomal alkylphosphocholines. Anti-Cancer Drugs. 1993;4:57-64.

35. Heerklotz H. Interactions of surfactants with lipid membranes. Q Rev Biophys. 2008;41:205-264.

36. Fleer EAM, Berkovic D, Unger C, Eibl H. Cellular uptake and metabolic-fate of hexadecylphosphocholine. Prog Exp Tumor Res. 1992;34:33-46.

37. Berger M, Sobottka S, Konstantinov SM, Eibl H. Erucylphosphocholine is the prototype of i.v. injectable alkylphosphocholines. Drugs today. 1998;34:73-81.

38. Lohmeyer M, Workman P. Growth arrest vs direct cytotoxicity and the importance of molecular structure for the in vitro antitumor activity of ether lipids. Brit J Cancer. 1995;72:277-286. 
39. Henke G, Lindner LH, Vogeser M, Eibl H-J, Woerner J, Mueller AC, Bamberg M, Wachholz K, Belka C, Jendrossek V. Pharmacokinetics and biodistribution of Erufosine in nude mice - Implications for combination with radiotherapy. Radiat Oncol. 2009;4:46.

40. Ríos-Marco P, Marco C, Cueto FJ, Carrasco MP, Jimenez-Lopez JM. Pleiotropic effects of antitumour alkylphospholipids on cholesterol transport and metabolism. Exp Cell Res. 2016;340:81-90.

41. Sobottka SB, Berger MR. Assessment of antineoplastic agents by MTT assay - Partial underestimation of antiproliferative properties. Cancer Chemother Pharmacol. 1992;30:385-393. 